\title{
DISCOVERY OF A LOW-MASS COMPANION TO A METAL-RICH F STAR WITH THE MARVELS PILOT PROJECT
}

\author{
Scott W. Fleming ${ }^{1}, \mathrm{Jian}_{\mathrm{Ge}}{ }^{1}$, Suvrath Mahadevan ${ }^{1,2,3}$ Brian Lee $^{1}$, Jason D. Eastman ${ }^{4}$, Robert J. Siverd ${ }^{4}$, \\ B. Scott Gaudi ${ }^{4}$, Andrzej Niedzielski ${ }^{5}$, Thirupathi Sivarani ${ }^{6}$, Keivan G. Stassun ${ }^{7}, 8$, Alex Wolszczan ${ }^{2,3}$, \\ Rory Barnes ${ }^{9}$, Bruce Gary ${ }^{7}$, Duy Cuong Nguyen ${ }^{1}$, Robert C. Morehead ${ }^{1}$, Xiaoke Wan $^{1}$, Bo Zhao ${ }^{1}$, Jian Liu ${ }^{1}$, \\ Pengcheng Guo ${ }^{1}$, Stephen R. Kane ${ }^{1,10}$, Julian C. van Eyken ${ }^{1,10}$, Nathan M. De Lee ${ }^{1}$, Justin R. CrepP ${ }^{1,11}$, \\ Alaina C. Shelden ${ }^{1,12}$, Chris Laws ${ }^{9}$, John P. Wisniewski ${ }^{9}$, Donald P. Schneider ${ }^{2,3}$, Joshua Pepper ${ }^{7}$, \\ Stephanie A. Snedden ${ }^{12}$, Kaike Pan ${ }^{12}$, Dmitry Bizyaev ${ }^{12}$, Howard Brewington ${ }^{12}$, Olena Malanushenko ${ }^{12}$, \\ Viktor Malanushenko ${ }^{12}$, Daniel Oravetz ${ }^{12}$, Audrey Simmons ${ }^{12}$, and Shannon Watters ${ }^{12,13}$ \\ ${ }^{1}$ Department of Astronomy, University of Florida, 211 Bryant Space Science Center, Gainesville, FL 326711-2055, USA; scfleming @ astro.ufl.edu \\ ${ }^{2}$ Department of Astronomy and Astrophysics, The Pennsylvania State University, 525 Davey Laboratory, University Park, PA 16802, USA \\ ${ }^{3}$ Center for Exoplanets and Habitable Worlds, The Pennsylvania State University, University Park, PA 16802, USA \\ ${ }^{4}$ Department of Astronomy, The Ohio State University, 140 West 18th Avenue, Columbus, OH 43210, USA \\ 5 Toruń Center for Astronomy, Nicolaus Copernicus University, ul. Gagarina 11, 87-100, Toruń, Poland \\ ${ }^{6}$ Indian Institute of Astrophysics, Bangalore 560034, India \\ ${ }^{7}$ Department of Physics and Astronomy, Vanderbilt University, Nashville, TN 37235, USA \\ ${ }^{8}$ Department of Physics, Fisk University, 1000 17th Ave. N., Nashville, TN 37208, USA \\ ${ }^{9}$ Department of Astronomy, University of Washington, P.O. Box 351580, Seattle, WA 98195, USA \\ ${ }^{10}$ NASA Exoplanet Science Institute, Caltech, MS 100-22, 770 South Wilson Avenue, Pasadena, CA 91125, USA \\ ${ }^{11}$ Department of Astronomy, California Institute of Technology, 1200 E. California Blvd., Pasadena, CA 91125, USA \\ 12 Apache Point Observatory, P.O. Box 59, Sunspot, NM 88349-0059, USA \\ ${ }^{13}$ Institute for Astronomy, 34 Ohia Ku St., Pukalani, HI 96768-8288, USA \\ Received 2010 April 26; accepted 2010 June 8; published 2010 July 13
}

\begin{abstract}
We report the discovery of a low-mass companion orbiting the metal-rich, main sequence F star TYC 2949-00557-1 during the Multi-object APO Radial Velocity Exoplanet Large-area Survey (MARVELS) pilot project. The host star has an effective temperature $T_{\text {eff }}=6135 \pm 40 \mathrm{~K}, \log g=4.4 \pm 0.1$, and $[\mathrm{Fe} / \mathrm{H}]=0.32 \pm 0.01$, indicating a mass of $M=1.25 \pm 0.09 M_{\odot}$ and $R=1.15 \pm 0.15 R_{\odot}$. The companion has an orbital period of 5.69449 \pm 0.00023 days and straddles the hydrogen burning limit with a minimum mass of $64 M_{J}$, and thus may be an example of the rare class of brown dwarfs orbiting at distances comparable to those of "Hot Jupiters." We present relative photometry that demonstrates that the host star is photometrically stable at the few millimagnitude level on time scales of hours to years, and rules out transits for a companion of radius $\gtrsim 0.8 R_{J}$ at the $95 \%$ confidence level. Tidal analysis of the system suggests that the star and companion are likely in a double synchronous state where both rotational and orbital synchronization have been achieved. This is the first low-mass companion detected with a multi-object, dispersed, fixed-delay interferometer.
\end{abstract}

Key words: brown dwarfs - planetary systems - stars: low-mass

Online-only material: color figures

\section{INTRODUCTION}

Studies of the frequency, parameter distributions, and correlations of extrasolar planets require homogeneous samples of hundreds of planets to obtain statistically significant results. Moreover, such a sample must have well-understood completeness limits, selection effects, and biases, which are easiest to obtain from a single, large-scale survey. Given current constraints on the frequency of giant planets, detection of such a large sample of planetary systems generally requires a precision radial velocity (RV) survey of many thousands of stars. Such a survey also provides a wealth of ancillary science. In particular, it is exquisitely sensitive to more massive companions, and because it targets a large and broad sample of host stars, it is naturally sensitive to rare binary systems in poorly explored regions of parameter space.

Of particular interest are the constraints on the frequency and parameter distributions of low-mass companions to solartype stars with masses near the hydrogen burning limit. One of the early results from precise RV searches was the apparent paucity of brown dwarf companions with minimum masses $\left(12 M_{J} \lesssim m \sin i \lesssim 80 M_{J}\right)$ at separations of $a \lesssim 5 \mathrm{AU}$, relative to more massive stellar companions and less massive planetary companions (Marcy \& Butler 2000). Note that we denote $i$ as the inclination angle between the companion's orbital angular momentum vector and the line of sight, and we reserve $I$ as the inclination angle of the stellar rotation axis to the line of sight. While the frequency of brown dwarf companions at larger separations is still relatively uncertain (e.g., Metchev \& Hillenbrand 2009), a meta-analysis of sets of known companions to solar-type stars by Grether \& Lineweaver (2006), with corrections for observational bias, confirmed the lack of brown dwarfs at close separations. These authors place the "driest" part of the brown dwarf desert at $\sim 20-50 M_{J}$, with a frequency of companions $\lesssim 0.5 \%$ in this range of masses.

Although there has been a steady increase in the number of known brown dwarf candidates via the RV technique (Marcy et al. 2001; Udry et al. 2002; Endl et al. 2004; Patel et al. 2007; Wittenmyer et al. 2009; Kane et al. 2009; Jenkins et al. 2009; Niedzielski et al. 2009; Omiya et al. 2009), most of these detections have been at separations $a \gtrsim 0.8 \mathrm{AU}$. Notable exceptions include the transiting brown dwarf CoRoT-Exo-3b 
with a period of $\sim 4$ days orbiting an F3V star (Deleuil et al. 2008), and HD41004Bb with a period of $\sim 1$ day orbiting the $\mathrm{M}$ dwarf component of a $\mathrm{K}-\mathrm{M}$ binary system (Santos et al. 2002). Brown dwarfs at such short orbital separations are of particular interest for several reasons. First, the frequency of such systems as a function of their physical and orbital parameters provide diagnostics that may be able to distinguish between the various mechanisms that have been invoked for their formation and dynamical evolution (e.g., Armitage \& Bonnell 2002; Matzner \& Levin 2005). In particular, these systems offer observational constraints on the poorly understood theory of tidal interactions between host stars and close companions (e.g., Mazeh 2008; Pont 2009). Second, these systems are much more likely to transit than their longer-period counterparts, as the transit probability is inversely proportional to orbital separation. Transiting systems yield valuable measurements on the masses, radii, and mean densities of brown dwarfs (Stassun et al. 2006, 2007; Deleuil et al. 2008).

Here we report the discovery of a candidate short-period, brown dwarf companion to the metal-rich star TYC 294900557-1, a main sequence $\mathrm{F}$ star with apparent brightness $V \sim 12.1$. This companion was discovered as part of the Multi-object APO Radial Velocity Exoplanet Large-area Survey (MARVELS) pilot project (hereafter MPP). The MPP used the W. M. Keck Exoplanet Tracker (Keck ET) instrument (Ge et al. 2006a) on the Sloan Digital Sky Survey (SDSS) $2.5 \mathrm{~m}$ telescope (Gunn et al. 2006) at the Apache Point Observatory. The Keck ET instrument is a multi-object (59 targets per exposure), dispersed fixed-delay interferometer (DFDI; Ge et al. 2002; Ge 2002; Erskine 2002; Erskine et al. 2003). In this instrument, fiber-fed starlight from the telescope is first passed through an iodine cell that acts as a stable wavelength reference. This light is then fed through a fixed-delay interferometer controlled via a piezoelectric transducer (PZT), and finally through a spectrograph that has a spectral resolution of $R=5100$. RV information is then imprinted in the phases of the fringes perpendicular to the dispersion axis of the spectrum due to a fixed variation in the interferometer delay along this direction.

The primary goal of the MPP was to demonstrate a fully integrated DFDI instrument capable of observing multiple stars in a single exposure. The second goal was to demonstrate that such an instrument is capable of achieving Doppler RV precision sufficient for extrasolar planet detection. The final goal was to formulate an operations procedure for conducting a survey using the Keck ET instrument in an efficient manner. These three goals were necessary preparations for the MARVELS (Ge et al. 2009) survey: a multi-object, DFDI, extrasolar planet survey that is part of Sloan Digital Sky Survey III (SDSS-III). ${ }^{14}$

The MARVELS pilot project was conducted in 2007, and consisted of 5-38 observations of 708 stars taken over 1-5 month baselines. Although the primary purpose of the MPP was to lay the groundwork for the full MARVELS survey, the cadence, number, and precision of the MPP was nevertheless sufficient to detect massive companions to a number of the target stars. The RV data for each star was initially fit using the RVSIM program (Kane et al. 2007). The companion to TYC 2949-00557-1 emerged as an excellent candidate, and here we describe the RV data, as well as additional spectroscopic and photometric data acquired to confirm the companion and further characterize the host star. TYC 2949-00557-1b is the first low-mass companion detected with a multi-object, dispersed, fixed-delay interferome-

\footnotetext{
14 http://www.sdss3.org/
}

Table 1

MPP RV Observations

\begin{tabular}{ccr}
\hline \hline BJD & $\begin{array}{c}\mathrm{RV} \\
\left(\mathrm{ms}^{-1}\right)\end{array}$ & $\begin{array}{r}\sigma_{\mathrm{RV}}^{\mathrm{a}} \\
\left(\mathrm{ms}^{-1}\right)\end{array}$ \\
\hline 2454101.69079 & 13339 & 94 \\
2454105.75520 & 19819 & 98 \\
2454106.70228 & 14981 & 96 \\
2454128.62211 & 19018 & 143 \\
2454128.86491 & 17170 & 91 \\
2454130.83623 & 13299 & 94 \\
2454136.64529 & 15043 & 85 \\
2454136.85894 & 15854 & 139 \\
2454163.72245 & 14882 & 91 \\
2454188.69749 & 21003 & 136 \\
2454191.68122 & 15421 & 97 \\
2454194.69216 & 21197 & 115 \\
2454195.68446 & 24453 & 102 \\
2454217.61115 & 22758 & 79 \\
\hline
\end{tabular}

Note. ${ }^{\text {a }}$ Errors are not scaled to account for systematics.

ter; previous observations with a single-object DFDI instrument at Kitt Peak National Observatory (KPNO) resulted in the first extrasolar planet discovered via this technique (Ge et al. 2006b), as well as the first confirmed planet via DFDI (van Eyken et al. 2004) and the ability to measure precise, absolute RVs with DFDI (Mahadevan et al. 2008).

\section{DOPPLER OBSERVATIONS}

\subsection{MPP Observations}

The MPP targeted $708 \mathrm{~F}, \mathrm{G}$, and $\mathrm{K}$ dwarfs with $7.6<V<12$ in 12 different fields, each containing 59 stars. Data for each field were processed simultaneously using a pipeline developed for multi-object DFDI instruments (see van Eyken et al. 2004; Ge et al. 2006b; Mahadevan et al. 2008, for details of basic DFDI processing steps.) For each target, we determine a "quality factor" (QF), which we define as

$$
\mathrm{QF}=\frac{\operatorname{rms}(X-\langle X\rangle)}{\operatorname{MEDIAN}\left(\sigma_{\mathrm{X}}\right)},
$$

where $X$ represents the RV measurements and rms is the rootmean-square residual. Targets that have $\mathrm{QF}>25$ either have intrinsically large RV variability or they are stars with lineof-sight rotational velocities $\gtrsim 10 \mathrm{~km} \mathrm{~s}^{-1}$, from which we are unable to extract precise RV measurements. TYC 2949-00557-1 was identified from a field with 58 other targets as having a QF of 37.20, in this case indicative of its binary nature. A total of 14 usable Doppler RV measurements were obtained spanning 116 days from 2007 January-April. Table 1 contains the barycentric Julian date using the Barycentric Dynamical Time standard (BJD $\left.{ }_{\mathrm{TDB}}\right)$, measured Doppler RV, and associated errors for all 14 observations. The Doppler velocities presented in Table 1 are absolute velocities calibrated to the solar spectrum, and are the sum of the barycentric velocity of the system and the additional Doppler variability caused by the companion. In order to provide an indication of the true level of systematic errors in the MPP data for objects in this field, we note that the 47 (likely constant) targets with $\mathrm{QF}<25$ in this field have a median quality factor of 3.25 .

We searched for periodicity in the Doppler data for TYC 2949-00557-1 using a Lomb-Scargle periodogram with floating mean (Lomb 1976; Scargle 1982; Cumming et al. 1999). 


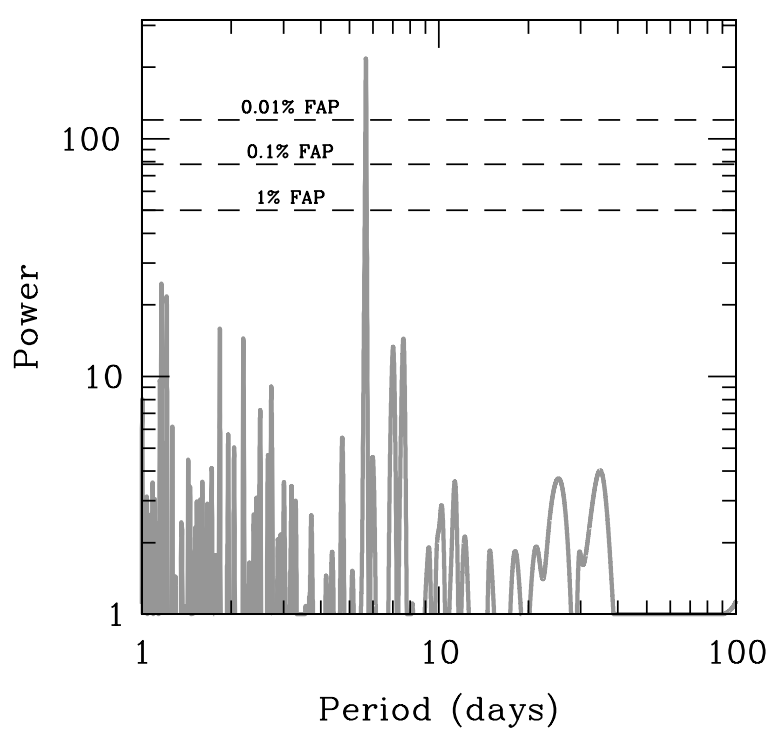

Figure 1. Lomb-Scargle periodogram for the MPP data (14 epochs). A clear and highly significant peak at $P=5.68$ days can be seen, with a power of $\sim 217$, which has a false alarm probability based on scrambling the data of $<0.01 \%$. Powers corresponding to false alarm probabilities of $1 \%, 0.1 \%$, and $0.01 \%$ are also shown

The resulting power spectrum is shown in Figure 1, revealing a clear peak at $P=5.68$ days with a power of $\sim 217$. To assess the significance of this peak, we ran a Monte Carlo simulation with $10^{5}$ trials. For each trial, we scrambled the times of the data points, computed the periodogram, and recorded the most significant peak. We found no trials with power greater than that of actual data, indicating a false alarm probability of $<10^{-5}$.

The advantage of a multi-object instrument is that the multiple targets that are observed simultaneously can be used to check for common systematic trends in data. Thus, as an additional check on the reliability of the observations, we constructed periodograms for the other 58 objects on the plate and calculated the power for each object at the period of the suspected companion. Any common systematics present in the data due to the sampling rate or instrumental effects will result in significant power at a common period for other targets. None of the other 58 targets have significant power at the period of TYC 2949-00557-1; the next strongest candidate has a false alarm probability (FAP) at that period of $77 \%$ and the other targets have FAPs $>99 \%$. The best-fit amplitude derived from the periodogram is $\sim 5500 \mathrm{~m} \mathrm{~s}^{-1}$, indicating a minimum mass in the brown dwarf regime for a solar-type primary. Given that brown dwarf companions in this period range are rare, we decided to obtain additional precise RV measurements, high-resolution spectra, absolute photometry, and precise relative photometry time series, in order to better characterize the primary and ascertain the nature of the companion.

\subsection{KPNO Doppler Observations}

Observations for the purpose of confirming the Doppler variability and orbit were conducted with the Exoplanet Tracker (ET) instrument (Ge et al. 2006b) at the KPNO with the $2.1 \mathrm{~m}$ telescope. Two observations separated by several hours were taken each night over seven consecutive nights starting on 2008 October 10. A total of 11 usable epochs were obtained. Integrations consisted of $60 \mathrm{~min}$ exposures bracketed by exposures of a Tungsten lamp passing through an iodine gas cell that acts as a calibration for instrument drift. Each arm of the interferometer
Table 2

KPNO/ET RV Observations

\begin{tabular}{ccc}
\hline \hline BJD & $\begin{array}{c}\mathrm{RV} \\
\left(\mathrm{ms}^{-1}\right)\end{array}$ & $\begin{array}{c}\sigma_{\mathrm{RV}}^{\mathrm{a}} \\
\left(\mathrm{ms}^{-1}\right)\end{array}$ \\
\hline 2454749.97070 & 266 & 58 \\
2454751.88336 & 1316 & 55 \\
2454751.94579 & 1985 & 50 \\
2454752.89657 & 7580 & 54 \\
2454752.95821 & 8026 & 58 \\
2454753.90161 & 9651 & 77 \\
2454753.96355 & 9548 & 65 \\
2454754.90388 & 4739 & 53 \\
2454754.96580 & 4502 & 52 \\
2454755.91284 & -694 & 47 \\
2454755.97519 & -996 & 52 \\
\hline
\end{tabular}

Note. ${ }^{a}$ Errors are not scaled to account for systematics.

produces a DFDI spectrum from which RVs are measured. The two beams are processed separately, and their measured RVs are combined via a weighted average based on the RV uncertainties. Table 2 contains the dates and velocities for the KPNO ET measurements. Unlike the results from the MPP, the velocities presented in Table 2 are relative RVs, i.e., measured relative to one of the epochs. Note that none of the velocities are exactly zero because an instrumental drift has been subtracted off based on the calibration lamps, and they are zeroed to a different epoch than the star. Because these data are relative RVs, an offset exists between the values in Tables 1 and 2 that must be included as an additional parameter when performing a combined analysis of the two data sets.

\subsection{HET Doppler Observations}

Observations of the candidate were also conducted using the $R=60,000$ mode of the high resolution spectrograph (HRS) (Tull 1998) on the Hobby-Eberly Telescope (HET) telescope (Ramsey et al. 1998) in the queue scheduled mode (Shetrone et al. 2007). The spectra consisted of 46 echelle orders recorded on the "blue" CCD detector (407.6-592 nm) and 24 orders on the "red" one (602-783.8 nm). The spectral data used for RV measurements were extracted from the 17 orders that cover the 505-592 nm range of the iodine spectrum. A total of 10 Doppler RV measurements were obtained spanning 83 days from 2008 December through 2009 February. The starlight was passed through an iodine cell to provide a stable reference to calibrate instrument drift. Two exposures without the iodine cell were taken to act as stellar templates. Due to the faintness of the target, the RVs were computed relative to each template and a mean value was determined. The results for both templates agree to within $3 \sigma$. Table 3 contains the dates and velocities for the HET measurements. Similar to the results from the KPNO ET in Table 2, these velocities are relative RVs, and therefore an offset exists between these values and the ones in both Tables 1 and 2 .

\subsection{Combined RV Analysis}

In order to check for consistency, we first fit the MPP, KPNO, and HET data sets individually to a seven parameter RV fit, where the seven parameters are the velocity semi-amplitude $K$, eccentricity $e$, argument of periastron $\omega$, period $P$, time of inferior conjunction of the companion $T_{c}$, velocity zero point $\gamma$, and linear slope $\dot{\gamma}$ (in order to allow for additional companions or systematic drifts). The best-fit solution was found using a 
Table 3

HET/HRS RV Observations

\begin{tabular}{crr}
\hline \hline BJD & $\begin{array}{c}\mathrm{RV} \\
\left(\mathrm{ms}^{-1}\right)\end{array}$ & $\begin{array}{r}\sigma_{\mathrm{RV}}^{\mathrm{a}} \\
\left(\mathrm{ms}^{-1}\right)\end{array}$ \\
\hline 2454807.74076 & 0 & 69 \\
2454808.98051 & 4674 & 67 \\
2454825.69507 & 2469 & 107 \\
2454829.70100 & 2482 & 66 \\
2454881.75734 & -23 & 286 \\
2454882.78369 & 3178 & 51 \\
2454883.76208 & 9451 & 63 \\
2454887.75592 & 165 & 48 \\
2454889.74685 & 10881 & 51 \\
2454890.75523 & 11513 & 47 \\
\hline
\end{tabular}

Note. ${ }^{\text {a }}$ Errors are not scaled to account for systematics.

hybrid downhill-simplex fit to the nonlinear parameters and an exact (linear) fit to the linear parameters. There are 14 MPP points, $11 \mathrm{KPNO}$ points, and $10 \mathrm{HET}$ points, so there are seven, four, and three degrees of freedom (dof), respectively.

For the MPP fit, we find a $\chi^{2} /$ dof of 44 . Given that the data points basically follow the model, and that the more precise HET data (whose error bars are overestimated, see below) fit the model well, the large $\chi^{2} /$ dof indicates that there are systematic uncertainties in addition to the photon noise, and thus the errors are severely underestimated. Given the large median $\mathrm{QF}=3.25$ found for the majority of the (likely constant) stars in this field, this result is not surprising. Indeed, there is a known systematic error in DFDI when utilizing an iodine cell in the stellar beam path (van Eyken et al. 2010). For the KPNO fit we find a $\chi^{2} /$ dof of 6.96 , once again indicating that the uncertainties are underestimated. For the HET fit, we find a $\chi^{2} /$ dof of 0.09 , therefore the uncertainties are likely overestimated for the HET data set. It is worth noting that a statistically significant slope is found when fitting the HET data. Fitting the HET data without a slope produces a significantly worse $\chi^{2} /$ dof.

We performed additional RV fitting to test the significance of the HET slope. Fitting the HET data with no slope and eccentricity forced to zero still results in a $\chi^{2} /$ dof $\ll 1$. Since this is the simplest model of an orbiting companion, it confirms that the HET uncertainties are overestimated. Several different models, in which slope is a free parameter, eccentricity is a free parameter, or both are free parameters, all result in lower $\chi^{2} /$ dof. There is no evidence of non-zero eccentricity in any of the best-fit solutions, but a significant slope is found in all cases when left as a free parameter. To be self-consistent, we allow for slopes in the MPP and KPNO data fitting as well, and note that their best-fit slopes are consistent with the HET value, but are much more poorly constrained due to the much larger RV uncertainties in those data sets. As a final check, we fit all data sets with eccentricity forced to zero and no slope, and find that the other orbital parameters are not qualitatively different from the result where slope is left as a free parameter. We therefore choose the case of zero eccentricity and non-zero slope as our preferred solution.

Uncertainties in the fitting parameters will be inaccurate if it is determined using misestimated RV errors. It is therefore important to attempt to correct the errors such that $\chi^{2} /$ dof $\sim 1$. However, given that we do not know why the errors are misestimated, particularly in the case of underestimated errors, the appropriate method to correct the uncertainties is not clear. Our approach was to try several different ways of correcting the errors to force $\chi^{2} /$ dof $=1$. Specifically, we investigated four different cases for treatment of the MPP (KPNO data come from a similar pipeline) and HET RV uncertainties: a scaling of the errors by a constant factor, an addition in quadrature of a constant error, a removal of suspected outliers based on the magnitude of the RV uncertainty followed by scaling, and a treatment of all data points with a constant error value. Ten Markov Chain Monte Carlo (MCMC) simulations were run for each case. The starting values for the parameters in these chains were chosen to span a range that is large with respect to the expected $1 \sigma$ uncertainty, the chains were stopped after reaching convergence as defined in Ford (2006), and then the chains were merged.

Analyzing the MPP and HET data separately yields discrepant periods at the $\sim 2 \sigma$ level for all cases of error treatment. We also found that the choice of error treatment can affect the derived value of $e \cos \omega$ from the MPP data as well at the $\sim 1 \sigma$ level. However, the other parameters from the MPP data, as well as all parameter values from the HET data, were not significantly affected by different treatments of the uncertainties. From this test, we conclude that there is no strong justification for removing any data points from the fit, so we conducted the final joint analysis of all three data sets where each set of errors is scaled by a constant factor.

The MPP errors are scaled by a factor of 6.64, the KPNO errors are scaled by 2.64 , and the HET errors are scaled by a factor of 0.3 such that the reduced $\chi^{2}$ is $\sim 1$ when each is fit independently. The fit including eccentricity as a free parameter is consistent with zero eccentricity, therefore we run a second fit that forces $e=0$. In that case, the error scalings are factors of $\{6.82,2.71,0.24\}$ for MPP, KPNO, and HET, respectively. We further scale the errors of all three data sets by a factor of 1.30 for the case where eccentricity is left as a free parameter, and 1.25 for the case where eccentricity is fixed to zero. This scaling is done so that the $\chi^{2} /$ dof $=1$ in the combined fit, and is necessary due to the systematics present in the data sets. Given the close separation of the companion, it is expected that the orbit has been tidally circularized, consistent with our findings. We therefore treat the case with eccentricity fixed at zero as our final values, but quote the parameters from both cases in Table 4, which contains the values of the orbital parameters for the case of non-zero eccentricity (eccentric) and eccentricity fixed at 0 (circular).

Figure 2 shows the final results of the joint RV fitting and fixing the eccentricity at 0. MPP data are the blue squares, KPNO data are the green triangles, HET data are the red circles, and the systemic velocity $\gamma_{0}$ has been removed. We find $\gamma_{0}=18.68 \pm 0.24 \mathrm{~km} \mathrm{~s}^{-1}$ for the star, with an offset between the MPP and KPNO data of $14.90 \pm 0.25 \mathrm{~km} \mathrm{~s}^{-1}$ and an offset between the MPP and HET data of $12.61 \pm 0.24 \mathrm{~km} \mathrm{~s}^{-1}$. The final orbital period is determined to be $5.69449 \pm 0.00023$ days and an RV semi-amplitude of $6.113 \pm 0.009 \mathrm{~km} \mathrm{~s}^{-1}$. We searched for an additional signal in the residuals from the joint fit that might be caused by an additional companion in the system, but found no other frequencies with significant power.

\section{RELATIVE TIME SERIES PHOTOMETRY OF THE HOST STAR}

Photometric observations are an important step in analyzing low-mass companions discovered via the Doppler technique. High-precision photometry can be used to search for transits of the companion. Additionally, time-series photometry can be 


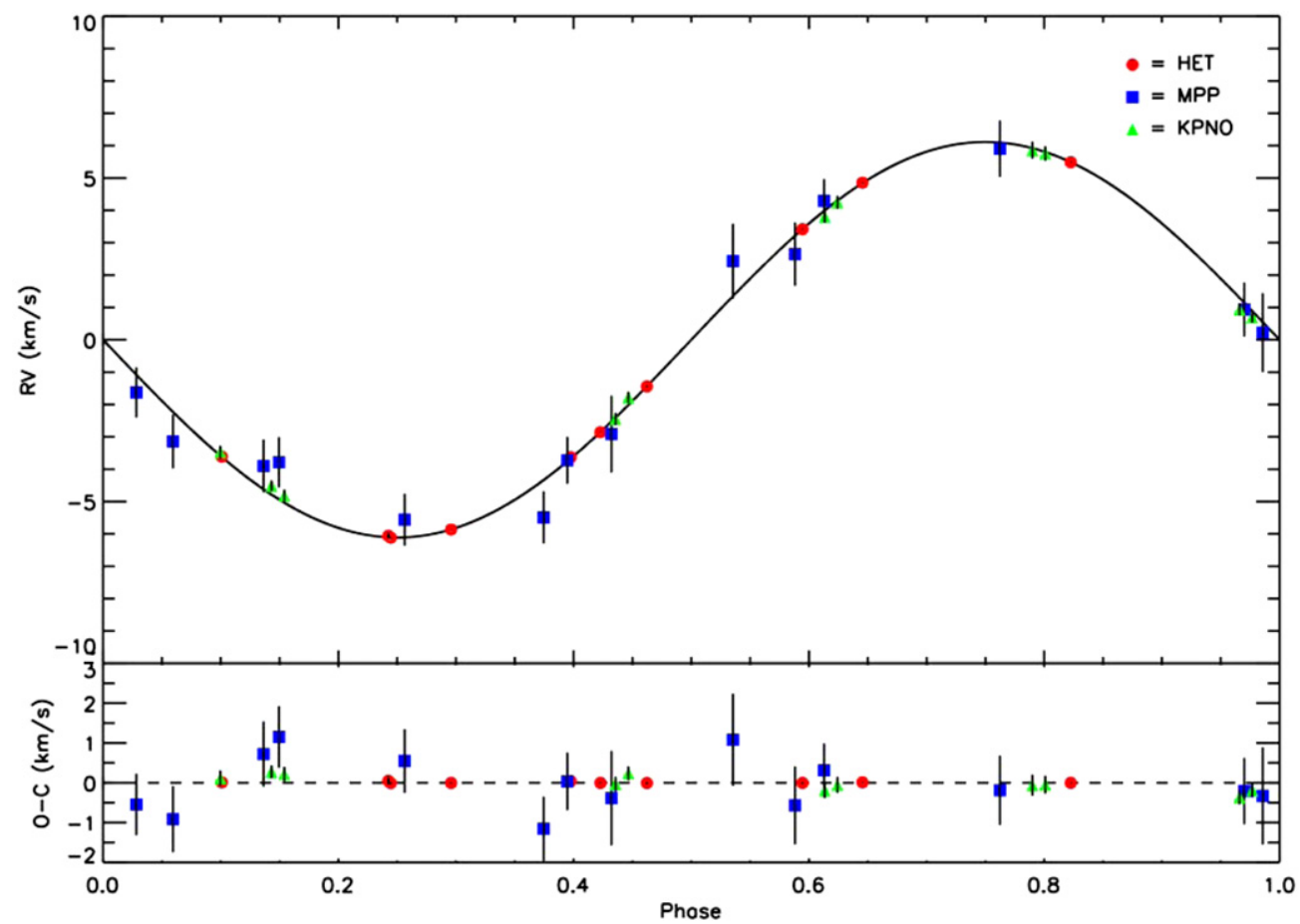

Figure 2. Results from the combined MPP, KPNO, and HET analysis, where the MPP errors are scaled by a factor of 8.49, KPNO errors are scaled by a factor of 3.37 , and the HET errors are scaled by a factor of 0.3 . The eccentricity is fixed at $e=0$. MPP data are in blue, KPNO data are in green, and HET data are in red. The systemic velocity $\gamma_{0}$ has been removed.

(A color version of this figure is available in the online journal.)

Table 4

Best-fit Dynamical Properties of TYC 2949-00557-1

\begin{tabular}{|c|c|c|c|c|}
\hline \multirow[t]{2}{*}{ Parameter } & Value & Uncertainty & Value & Uncertainty \\
\hline & \multicolumn{2}{|c|}{ Eccentric Case } & \multicolumn{2}{|c|}{ Circular Case } \\
\hline Period (days) & 5.69459 & 0.00029 & 5.69449 & 0.00023 \\
\hline $\mathrm{K}\left(\mathrm{km} \mathrm{s}^{-1}\right)$ & 6.109 & 0.014 & 6.113 & 0.009 \\
\hline$T_{c}\left(\mathrm{BJD}_{\mathrm{TDB}}-2454000\right)$ & 868.9878 & 0.0042 & 868.9877 & 0.0016 \\
\hline$e \cos \omega$ & 0.0000 & 0.0015 & 0. & $\ldots$ \\
\hline$e \sin \omega$ & -0.0005 & $\begin{array}{l}+0.0011 \\
-0.0019\end{array}$ & 0. & $\ldots$ \\
\hline$e$ & 0.0017 & $\begin{array}{l}+0.0019 \\
-0.0017\end{array}$ & 0. & $\cdots$ \\
\hline$\omega(\mathrm{rad})$ & 4.69 & 1.58 & $\frac{\pi}{2}$ & $\cdots$ \\
\hline$m_{\min } i\left(\mathrm{M}_{J}\right)$ & 64.3 & 3.0 & 64.3 & 3.0 \\
\hline Systemic velocity $\gamma_{0}\left(\mathrm{~km} \mathrm{~s}^{-1}\right)$ & 18.67 & 0.26 & 18.68 & 0.24 \\
\hline KPNO offset $\left(\gamma_{0}-\gamma_{\text {kpno }}, \mathrm{km} \mathrm{s}^{-1}\right)$ & 14.89 & 0.26 & 14.90 & 0.25 \\
\hline HET offset $\left(\gamma_{0}-\gamma_{\text {het }}, \mathrm{km} \mathrm{s}^{-1}\right)$ & 12.59 & 0.26 & 12.61 & 0.24 \\
\hline MPP slope $\dot{\gamma}_{\mathrm{mpp}}\left(\mathrm{km} \mathrm{s}^{-1} \mathrm{day}^{-1}\right)$ & -0.0044 & 0.0065 & -0.0037 & 0.0065 \\
\hline KPNO slope $\dot{\gamma}_{\mathrm{kpno}}\left(\mathrm{km} \mathrm{s}^{-1} \mathrm{day}^{-1}\right)$ & 0.016 & 0.034 & 0.013 & 0.033 \\
\hline HET slope $\dot{\gamma}_{\text {het }}\left(\mathrm{km} \mathrm{s}^{-1}\right.$ day $\left.^{-1}\right)$ & -0.0010 & 0.00035 & -0.0011 & 0.00024 \\
\hline Total $\sigma_{\mathrm{RV}}$ Scale Factor (MPP) & 8.64 & $\ldots$ & 8.49 & $\ldots$ \\
\hline Total $\sigma_{\mathrm{RV}}$ Scale Factor (KPNO) & 3.43 & $\ldots$ & 3.37 & $\ldots$ \\
\hline Total $\sigma_{\mathrm{RV}}$ Scale Factor (HET) & 0.39 & $\ldots$ & 0.30 & $\ldots$ \\
\hline Combined $\chi^{2} /$ dof & 1.69 & $\ldots$ & 1.55 & $\ldots$ \\
\hline
\end{tabular}

used to rule out stellar mechanisms of Doppler variability, such as chromospheric activity due to starspots or stellar pulsations. In the case of stars with detectable starspots, time-series photometry can be used to determine a stellar rotation rate. In this section, we present and analyze time series relative photometry of TYC-2949-00557-1 from two sources: relatively precise (few mmags) photometry covering a relatively short timespan $(2-8 \mathrm{hr}$ over five nights) from the Hereford Arizona Observatory, and less precise (few percent), but more comprehensive photometry consisting of 7194 epochs taken over roughly three years as part of the Kilodegree Extremely Little Telescope (KELT) North transit survey. Neither data sets show any evidence for variability of the host star.

\subsection{Relative Photometry from Hereford Arizona Observatory}

Initial photometric observations of the primary were performed on four nights in 2009 (February 19, 21, and 27, and March 16) at the Hereford Arizona Observatory (observatory code G95 in the IAU Minor Planet Center), a private facility in southern Arizona. Additional observations were made in 2010 on April 15 to search for transits based on an updated transit ephemeris from the combined RV analysis (Section 2.4). All data were taken with an 11 inch Celestron Schmidt-Cassegrain (model CPC 1100) telescope that is fork-mounted on an equatorial wedge, an SBIG ST-8XE CCD with a KAF 1602E detector, and an SBIG AO-7 tip-tilt image stabilizer used to maintain the field at a fixed position on the CCD. The observations in 2009 were done without a filter (" $C$ " band), resulting in an effective central wavelength of $\sim 570 \mathrm{~nm}$ between Johnson $V$ and $R$ bands. The observations in 2010 were taken with a Sloan $r^{\prime}$ filter. Data toward the end of the night on 2010 April 15 were taken at very high air mass (out to $\sec z=5.7$ ), resulting is somewhat degraded photometric precision.

Figure 3 shows the relative photometry over the five nights, which demonstrate that the primary star is intrinsically stable on the time scale of several hours, at the level of 2-4 millimagnitudes. Based on the final ephemerides determined in Section 2.4, only the last night (2010 April 15, top row) covers possible times of predicted transits. The vertical bars are the predicted times of ingress, mid-transit, and egress based on the RV fit in Section 2.4 for the two methods of RV fitting (" $C$ " is for $e=0$, " $E$ " is for non-zero eccentricity), and an assumed transit duration of $3.3 \mathrm{hr}$, corresponding to a nearly central transit. The widths 
TYC 2949-00557-1, Hereford Arizona Observatory

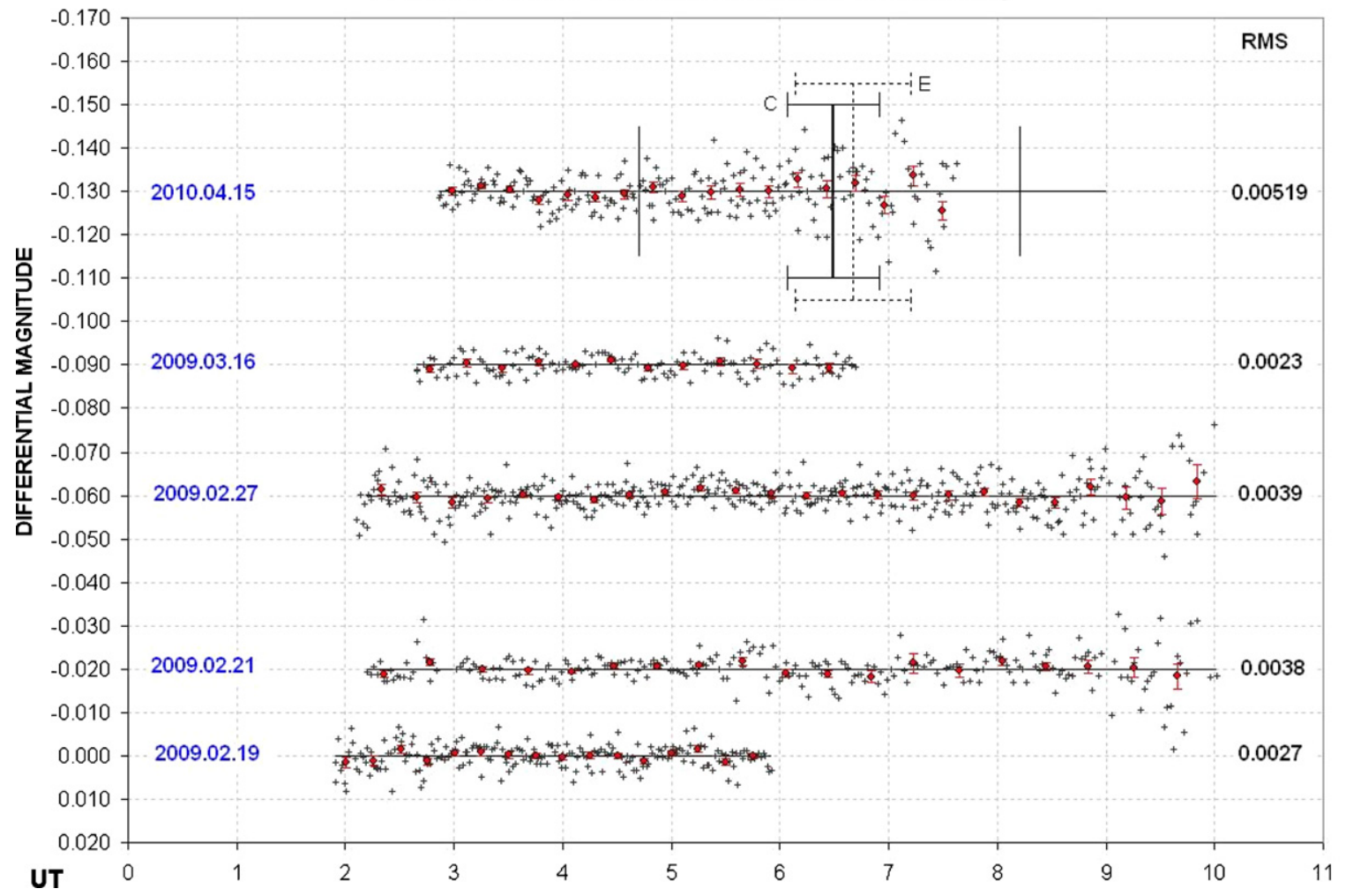

Figure 3. Photometric observations with the Hereford Arizona Observatory telescope. The horizontal axis is elapsed time each night in hours. The star is photometrically stable with an rms of 2-4 mmag. The increased scatter towards the end of 2009 February 21, 2009 February 27, and most of 2010 April 15 are due to observing at high air mass. The vertical bars are the predicted times of ingress, mid-transit and egress based on the RV fit in Section 2.4 and an assumed transit duration of $3.3 \mathrm{hr}$. The two mid-transit estimates are based on the two results from the RV fitting (" $C$ " is for $e=0$, " $E$ " is for the case where $e$ is left as a free parameter). The widths correspond to the uncertainties in the mid-transit times.

(A color version of this figure is available in the online journal.)

correspond to the uncertainties in the mid-transit times. There is no evidence of a transit at the most likely depth $(\sim 0.8 \%)$ and duration during these observations. In Section 3.3, we consider the uncertainties in the ephemeris and properties of the primary, as well as a range of impact parameters, to quantify the confidence with which we can exclude transits in this system.

\subsection{Relative Photometry with KELT}

KELT North survey is a wide-field photometric survey of $\sim 40 \%$ of the northern sky designed to monitor fairly bright $(8<V<12)$ stars in order to search for planetary transits (Pepper et al. 2003, 2007; Siverd et al. 2009). The KELT survey instrument consists of an Apogee AP16E $(4 \mathrm{~K} \times 4 \mathrm{~K} 9 \mu \mathrm{m}$ pixels $)$ CCD camera attached to a Mamiya 645 medium-format $42 \mathrm{~mm}$ aperture camera lens. The resultant field of view is $26^{\circ} \times 26^{\circ}$ at roughly $23^{\prime \prime}$ pixel $^{-1}$. The standard configuration uses a Kodak Wratten 8 red-pass filter and the resultant bandpass resembles a widened Johnson-Cousins $R$ band. KELT-N is permanently mounted on a fixed pier at Winer Observatory in Sonoita, AZ.

The KELT-N survey targets 13 star fields centered at 31.7 declination (the survey site latitude) spaced fairly evenly through all $24 \mathrm{hr}$ of R.A. with slight overlap. Exposure times are $150 \mathrm{~s}$, which yields relative photometric precisions of better than a few percent for $V \lesssim 12$. Typical cadences are roughly 20 to 30 minutes when the target field is visible, and to date there exist $\sim 5000-7000$ epochs per target. The areal sky coverage, target magnitude range, and high photometric precision of the KELT survey results in excellent synergy with the MPP (as well as the full MARVELS survey). TYC 2949-00557-1 is in one of KELT's target fields and is a good example of this synergy. We use the KELT photometry to characterize the photometric variability of the host star, and to search for signatures of transits of the companion. We first briefly describe the data reduction, and then describe the light curve analysis.

Images of the field are flat-fielded, and then relative photometry is extracted using the ISIS image subtraction package (Alard \& Lupton 1998), in combination with DAOPHOT (Stetson 1987) to perform point-spread function fitting photometry. We further eliminate problematic images due to poor observing conditions by examining outliers from the ensemble of individual light curves on the CCD. Any epochs that produce photometric outliers in a significant fraction $(\gtrsim 5 \%)$ of light curves are removed from all light curves. We use the VARTOOLS program (Hartman et al. 2008) to remove common trends due to systematic errors from the light curves using the Trend Filtering Algorithm (Kovács et al. 2005), first removing the 20 points with the highest flux and the 20 points with the lowest flux from all light curves. We choose the 400 stars with the lowest rms values as comparison stars for trend removal, ensuring that these stars are evenly distributed across the region of the CCD near the target, and excluding variable stars and saturated stars. Finally, the errors of all the light curves are scaled by a constant value, chosen to force the modal value of $\chi^{2} /$ dof for a weighted, constant flux fit to the light curves to be unity for the ensemble. For TYC 2949-00557-1, this procedure resulted in a $\chi^{2} /$ dof that differed somewhat $(\lesssim 50 \%)$ from unity. Since 

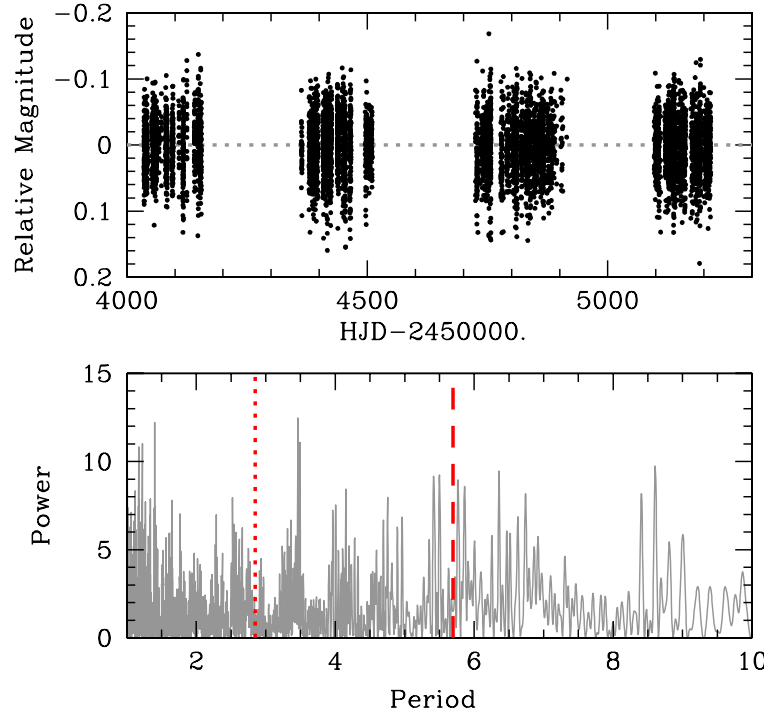

Figure 4. Top panel: KELT-N light curve for TYC 2949-00557-1. Bottom panel: Lomb-Scargle periodogram of the KELT data, showing no evidence for any significant periodicities for periods of $P=1-10$ days, including the period of the RV companion (vertical dashed line) and the first harmonic (vertical dotted line)

(A color version of this figure is available in the online journal.)

we see no evidence for photometric variability for this star (see below) we further scale the errors to force $\chi^{2} /$ dof $=1$ to be conservative. TYC 2949-00557-1 happened to fall in an overlap region of two target fields, and as a result we had two sets of light curves for the target. These data were reduced independently, and then combined after subtracting the difference between their weighted mean magnitudes.

The KELT light curve for TYC 2949-00557-1 is shown in Figure 4. It contains 7194 data points spanning 3.2 years, and has a weighted rms of $3.5 \%$. We search for variability using a weighted Lomb-Scargle periodogram with floating mean (Lomb 1976; Scargle 1982; Cumming et al. 1999), and find no significant peaks (see Figure 4), and in particular no evidence for periodic variability near the period of the companion ( $P \simeq 5.69$ days), or the first harmonic $(P / 2)$. Figure 5 shows the KELT lightcurve, phased according to the best-fit RV ephemeris (Table 4), as well as binned 0.025 in phase (3.46 hr). The rms of the binned data is $\sim 2.4 \mathrm{mmag}$, and the $\chi^{2} / \mathrm{dof}=0.91$ for a constant fit, indicating a low level of correlated noise, and no evidence for variability at the few mmag level. We limit the amplitude of any variability at $P / 2$ to be $\lesssim 2 \mathrm{mmag}$; unfortunately this is well above the level of ellipsoidal variability expected for this companion of $\sim 0.03 \sin i$ mmag (Pfahl et al. 2008).

\subsection{Limits on Transits}

Given the relatively high a priori transit probability of TYC 2949-00557-1b of $\sim R / a \sim 8 \%$, where $R$ is the stellar radius, we searched for transits in the KELT dataset combined with the Hereford data from 2010 April 15 . The expected transit duration is $\sim R P /(\pi a) \sim 3.4 \mathrm{hr}$ for a central transit, and the expected fractional depth is $\delta \sim(r / R)^{2} \sim 0.8 \%\left(r / R_{J}\right)^{2}$, where $r$ is the radius of the companion. The expected radius of the companion depends on its true mass, as well as the age of the system, but is likely to be $\sim 1 R_{J}$ (Baraffe et al. 2003). Unfortunately, while the KELT data has excellent phase coverage, it is not of sufficient quality to detect or rule out the expected signal. If transits were present, we should expect to

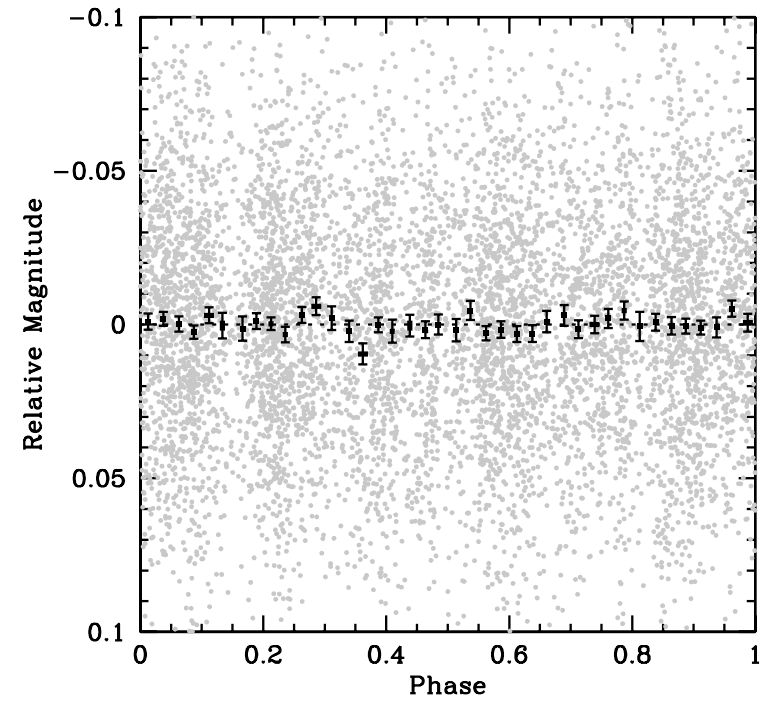

Figure 5. Small filled circles show the KELT-N light curve for TYC 294900557-1 phased according to the ephemeris from the joint RV fit (see Section 2.4). The larger filled squares with error bars are binned 0.025 in phase.

detect them with a signal-to-noise ratio of

$$
\mathrm{S} / \mathrm{N} \sim N^{1 / 2}\left(\frac{R}{\pi a}\right)^{1 / 2} \frac{\delta}{\sigma}
$$

where $N=7194$ is the number of data points, and $\sigma \sim 3.5 \%$ is the typical uncertainty. Thus, $\mathrm{S} / \mathrm{N} \sim 3\left(r / R_{J}\right)^{2}$, which is marginal unless the companion has a radius significantly larger than Jupiter. On the other hand, the majority of the Hereford data is generally of sufficient quality to detect transits at the expected depth, and one night covers the predicted transit time for the companion. Unfortunately, there is no indication of a transit at the expected time.

We nevertheless proceed with a quantitative search for a transit signal. We combine the KELT data with the Hereford data from 2010 April 15 after first subtracting the difference between their weighted mean magnitudes. The slight difference in passband between the HAO and KELT data do not affect the ability to detect a transit in the combined data set. We use the distribution of companion periods $P$ and expected transit times $T_{c}$ from the MCMC analysis of the combined RV data described in Section 2.4. For each combination of $T_{c}$ and $P$ (i.e., for each link in the Markov Chain), we draw a random value for the $T_{\text {eff }}, \log g$, and $[\mathrm{Fe} / \mathrm{H}]$ of the primary from a Gaussian distribution, with the central values and dispersions given in Table 5, as determined from the spectroscopic analysis described in Section 5.2. We then use the Torres et al. (2010) empirical relations to estimate the mass $M$ and radius $R$ of the primary for those values. We add an additional offset to $M$ and $R$ drawn from Gaussians with dispersions equal to the dispersions of the fits to the empirical relations in Torres et al. (2010); specifically $6.4 \%$ in $M$ and $3.2 \%$ in $R$. Finally, we draw a random value of the impact parameter of the transit in units of the radius of the star in the range $[0,1]$. Assuming a radius for the companion, we then compute the expected transit curve using the routines of Mandel \& Agol (2002), using limb-darkening coefficients from Claret (2000), assuming that both the KELT and Hereford bandpasses roughly correspond to $R$. We then fit this curve to the combined data set, and compute the improvement in $\chi^{2}$ relative to a constant flux fit to the data. We repeat this for each link in the Markov chain, as well as for a variety of different companion radii. 
Table 5

Stellar Host Properties of TYC 2949-00557-1 ${ }^{\mathrm{a}}$

\begin{tabular}{lcc}
\hline \hline \multicolumn{1}{c}{ Parameter } & Value & Uncertainty \\
\hline$\alpha(\mathrm{J} 2000)^{\mathrm{b}}$ & $101.921152(\mathrm{deg})$ & $06: 47: 41.076(\mathrm{HH}: \mathrm{MM}: \mathrm{SS})$ \\
$\delta(\mathrm{J} 2000)^{\mathrm{b}}$ & $42.009332(\mathrm{deg})$ & $42: 00: 33.60$ (DD:MM:SS) \\
$B$ & 12.846 & 0.023 \\
$V$ & 12.142 & 0.031 \\
$R_{C}$ & 11.750 & 0.039 \\
$I_{C}$ & 11.391 & 0.043 \\
$J_{2 \mathrm{MASS}}$ & 10.820 & 0.022 \\
$H_{2 \mathrm{MASS}}$ & 10.474 & 0.021 \\
$K_{2 \mathrm{MASS}}$ & 10.421 & 0.018 \\
$T_{\text {eff }}(\mathrm{K})$ & 6135. & 40. \\
$\log \left(g\left[\mathrm{~cm} \mathrm{~s}^{-1}\right]\right)$ & 4.4 & 0.1 \\
{$[$ Fe $/ \mathrm{H}]$} & 0.32 & 0.01 \\
$v$ sin $I\left(\mathrm{~km} \mathrm{~s}^{-1}\right)$ & 7. & 1. \\
$M_{\text {primary }}\left(M_{\odot}\right)$ & 1.25 & 0.09 \\
$R_{\text {primary }}\left(R_{\odot}\right)$ & 1.15 & 0.15 \\
$d(\mathrm{pc})$ & 413 & +66 \\
& & -57 \\
\hline
\end{tabular}

Notes.

${ }^{a}$ BVRI magnitudes are unweighted averages from Table 6.

b Coordinates taken from the Tycho 2 Catalog (Høg et al. 2000)

We search for significant improvements in $\chi^{2}$ which would be indicative of a detection. Our best-fit has $\Delta \chi^{2}=-11.7$ relative to a constant flux fit. In order to asses the significance of this improvement in $\chi^{2}$, we repeat the search for "anti-transit," i.e., signals with the same shape as a transit but corresponding to an increase in flux (see Burke et al. 2006), and find improvements in $\chi^{2}$ at similar levels. We therefore conclude that there is no evidence for a transit in the combined KELT and Hereford data.

Given that we have not detected any evidence of transits, we now ask what the probability is that we would have detected a transit of a given radius, assuming that the companion transits (i.e., $b \leqslant 1$ ). To do this, we simply determine what fraction of the steps in the Markov Chain described above result in an increase in $\chi^{2}$ above a certain level, as a function of the radius of the companion. This result is shown in Figure 6 , for $\Delta \chi^{2}=$ $\{9,16,25\}$. The $\Delta \chi^{2}$ values were chosen as representative values: $\Delta \chi^{2}=16$ is the likely detection limit, 25 is chosen as a conservative limit, and 9 is chosen to straddle the true detection limit. The black, long-dashed line is a case where flatbottomed, boxcar-shaped transits (no ingress/egress) were used and represents $\Delta \chi^{2}=16$. It shows that detailed modeling of limb darkening and the ingress/egress has little effect on the final results of this test. Given the properties of the noise as estimated from the improvements in the fits from "antitransits," signals with $\Delta \chi^{2} \gtrsim 16$ are likely to have been reliably detected. Thus, we can exclude transits of a companion with $r \gtrsim 0.75 R_{J}$. at the $95 \%$ confidence level. Baraffe et al. (2003) predict radii of $\gtrsim 0.77 R_{J}$ for brown dwarfs with $m \simeq 60 M_{J}$ for ages of $\lesssim 5$ Gyr. We conclude it is unlikely this companion transits, unless the system is substantially older than 5 Gyr, which is unlikely given the effective temperature and surface gravity of the host star (Section 6).

\section{ABSOLUTE PHOTOMETRY}

The Tycho-2 catalog's (Høg et al. 2000) V-band magnitude for this object is 11.840 , however, Tycho magnitudes are known to significantly degrade beyond $V_{T}>11$. Measurements were taken at Hereford Arizona Observatory using both $B, V, R_{c}, I_{c}$ and Sloan $g^{\prime}, r^{\prime}, i^{\prime}$ filter sets. A total of 64 Landolt standard

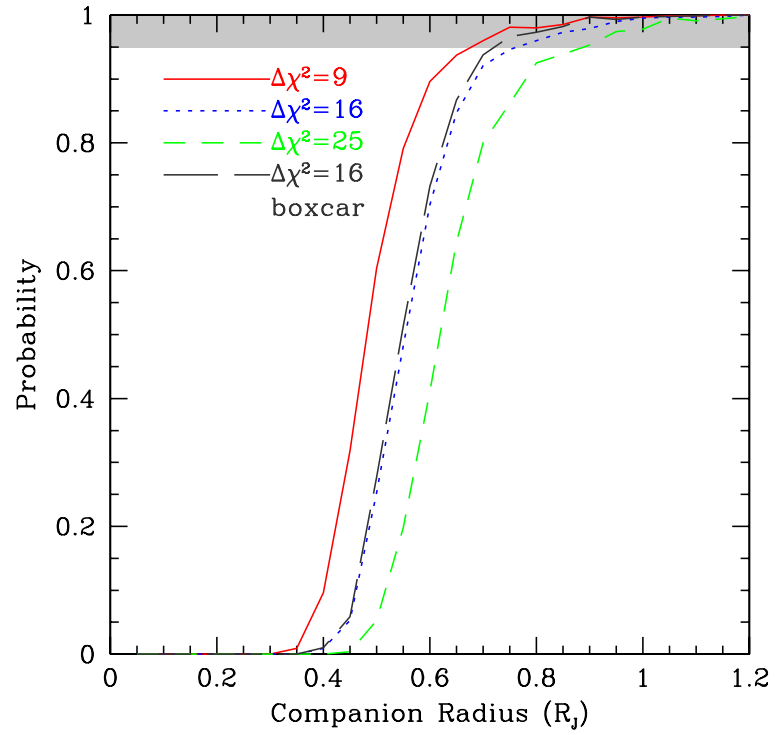

Figure 6. Probability that transits of a companion are excluded at levels of $\Delta \chi^{2}=\{9,16,25\}$ based on the analysis of the combined Hereford and KELT photometric data sets, as a function of the radius of the companion. The black, long-dashed line is a case where boxcar-shaped transits were used as a test, and is for $\Delta \chi^{2}=16$. Transits of companions with radius $r \gtrsim 0.75 R_{J}$ can be excluded at the $95 \%$ confidence level.

(A color version of this figure is available in the online journal.)

Table 6

Measured Photometry for TYC 2949-00557-1 from Hereford Arizona Observatory

\begin{tabular}{lcc}
\hline \hline Band $^{\mathrm{a}}$ & $\begin{array}{c}\text { Flux } \\
(\mathrm{mag})\end{array}$ & $\begin{array}{c}\text { Uncertainty } \\
(\mathrm{mag})\end{array}$ \\
\hline$B$ & 12.839 & 0.023 \\
$V$ & 12.135 & 0.031 \\
$R c$ & 11.750 & 0.039 \\
$I c$ & 11.390 & 0.043 \\
$g^{\prime}$ & 12.450 & 0.020 \\
$r^{\prime}$ & 11.937 & 0.016 \\
$i^{\prime}$ & 11.811 & 0.028 \\
$B_{\text {Sloan }}$ & 12.853 & 0.023 \\
$V_{\text {Sloan }}$ & 12.150 & 0.024 \\
$R c_{\text {Sloan }}$ & 11.749 & 0.029 \\
$I_{\text {Sloan }}$ & 11.392 & 0.043 \\
\hline
\end{tabular}

Note. ${ }^{\text {a }}$ Measurements labeled with Sloan are converted from the Sloan filter observations using Smith et al. (2002) transformation equations.

stars and 15 SDSS standard stars were used as calibrators. The conversion equations of Smith et al. (2002) were used to convert the Sloan filter measurements into the BVRI system. The agreement between the observed $B V R I$ magnitudes and those converted from Sloan magnitudes agree within the measurement error. We adopt the unweighted average of each $B V R I$ measurement and the larger of the two statistical errors for the final magnitude results. Table 6 summarizes the measured magnitudes and final results for the multi-band photometry. Near-IR fluxes are taken from the 2MASS (Skrutskie et al. 2006) Point Source Catalog and are presented in Table 5.

\section{CHARACTERIZATION OF THE HOST STAR}

\subsection{SED Analysis}

We use the BVRI fluxes along with the 2MASS near-IR data to fit model spectral energy diagrams (SEDs) to derive 

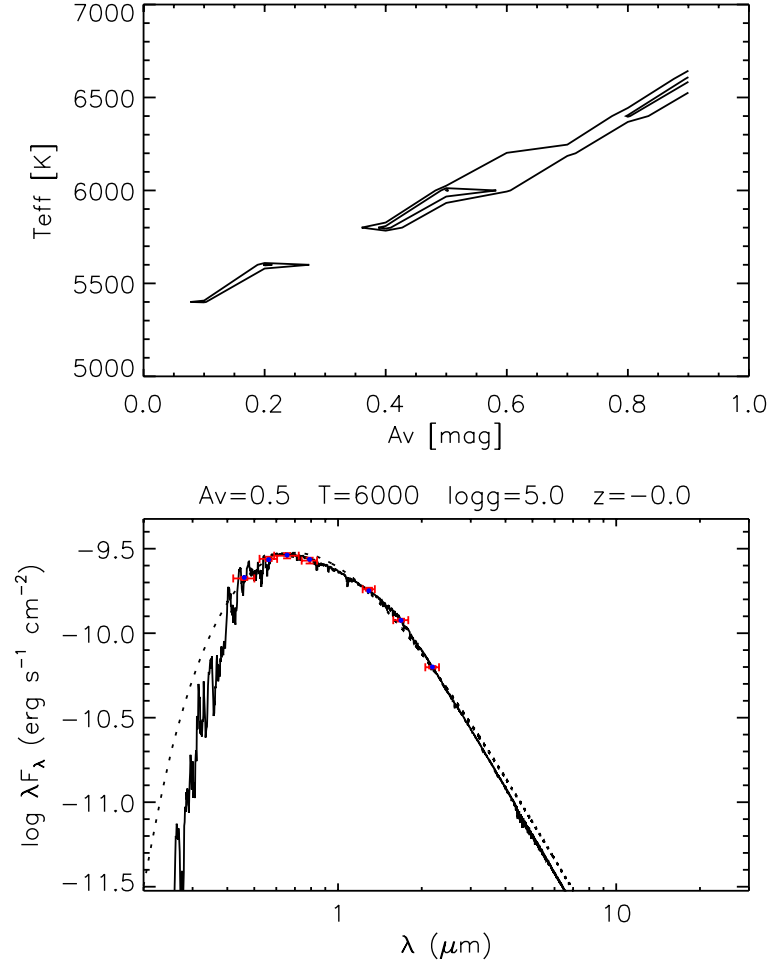

Figure 7. Top: $\chi^{2}$ map in $T_{\mathrm{eff}}-A_{V}$ space showing the degeneracy between extinction and $T_{\text {eff }}$. Inner contours are $1 \sigma$ uncertainties for $T_{\text {eff }}$ and $A_{V}$ assuming they are the only significant degrees of freedom, outer contours assume all four parameters are significant. Bottom: NextGen model overplotted on the observed fluxes. The dotted curve is a blackbody SED for the best-fit $T_{\text {eff }}$.

(A color version of this figure is available in the online journal.)

approximate stellar parameters. NextGen models from Hauschildt et al. (1999) in grids of $100 \mathrm{~K}$ for $T_{\text {eff }}, 0.5 \mathrm{dex}$ for $\log g$ and $0.5 \mathrm{dex}$ for the metallicity, represented by $[\mathrm{Z} / \mathrm{H}]$, are fit along with the line-of-sight extinction. There is a degeneracy between line-of-sight extinction and derived $T_{\text {eff }}$ when fitting SEDs with unknown extinction. Figure 7 (top) shows the $\chi^{2}$ map in $T_{\text {eff }}-A_{V}$ space. The interior contours represent $1 \sigma$ uncertainties assuming that $T_{\text {eff }}$ and $A_{V}$ are the only two free parameters. The exterior contours are the $1 \sigma$ uncertainties with all four parameters $\left(T_{\text {eff }}, A_{V}, \log g\right.$ and $[\mathrm{Z} / \mathrm{H}])$ as free parameters. We find the global minimum in $\chi^{2}$-space is for a $T_{\text {eff }}=6000 \mathrm{~K}, \log g$ of $5.0,[\mathrm{Z} / \mathrm{H}]$ of 0.0 and extinction $A_{V}=0.5$. Figure 7 (bottom) shows the best-fit NextGen model along with the observed fluxes. Other solutions exist within the $1 \sigma$ contours at cooler $T_{\text {eff }}$ and smaller $A_{V}$; however, the dust maps of Schlegel et al. (1998) give an $A_{V}$ of 0.45 along this line of sight, consistent with the best-fit $A_{V}$ of 0.5 assuming that the star is behind the majority of the dust along this line of sight. The hotter temperature solution is also consistent with the $T_{\text {eff }}$ derived from Echelle spectra presented in Section 5.2.

\subsection{Spectral Synthesis}

In order to derive physical properties of the host star and estimate the minimum mass of the companion, stellar templates from the HET observations that do not contain iodine lines were used to derive parameters of the host star. We use the latest MARCS model atmospheres (Gustafsson et al. 2008) for the analysis. Generation of synthetic spectra and the line analysis were performed using the turbospectrum code (Alvarez \& Plez 1998), which employs line broadening accord-

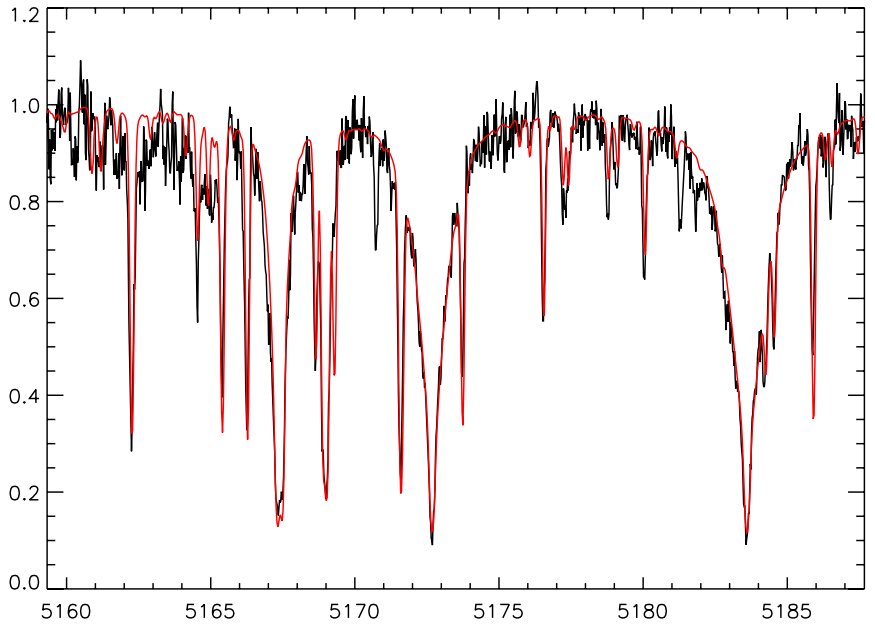

Figure 8. HET/HRS template spectrum in black with best-fit model in red for the $\mathrm{Mgb}$ region. The spectra have been continuum normalized and the relative flux density is plotted against the wavelength in Angstroms.

(A color version of this figure is available in the online journal.)

ing to the prescription of Barklem \& O'Mara (1998). The line lists used are drawn from a variety of sources. Updated atomic lines are taken mainly from the Vienna Atomic Line Database (VALD) database (Kupka et al. 1999). The molecular species $\mathrm{CH}, \mathrm{CN}, \mathrm{OH}, \mathrm{CaH}$, and $\mathrm{TiO}$ are provided by B. Plez (see Plez \& Cohen 2005), while the $\mathrm{NH}, \mathrm{MgH}$, and $\mathrm{C} 2$ molecules are from the Kurucz linelists. The solar abundances used here are the same as Asplund (2005). The Fe I and Fe II lines used for the line analysis were compiled by Santos et al. (2004), who use solar Fe abundance to derive gf values. Both Asplund (2005) and Santos et al. (2004) use HARPS solar spectra and an Fe abundance of 7.45 .

We derive a $T_{\text {eff }}=6135 \pm 40 \mathrm{~K}$ and $[\mathrm{Fe} / \mathrm{H}]=0.32 \pm 0.01$, based on $\mathrm{Fe}$ I excitation equilibrium and a $\log g=4.4 \pm 0.1$ based on the ionization equilibrium of $\mathrm{Fe} \mathrm{I}$ and $\mathrm{Fe}$ II lines and by fitting the wings of the Mgb triplet at 5167, 5172 and $5183 \AA$. The error estimates are based on the equivalent width of $\mathrm{Fe}$ lines and the errors of $\mathrm{Fe}$ abundances from the individual lines. A microturbulence value $\xi_{t}=1.65 \mathrm{~km} \mathrm{~s}^{-1}$ is derived by forcing weak and strong Fe I lines to give the same abundances. Fitting the Fe lines in the $\mathrm{Mgb}$ region yields a rotational velocity of $v \sin I=7 \mathrm{~km} \mathrm{~s}^{-1}$. We only used the $110 \mathrm{Fe}_{\mathrm{I}}$ lines weaker than $100 \mathrm{~m} \AA$ for the analysis. Figure 8 shows the continuum normalized spectra in black and the best-fit model in red for the Mgb region.

In addition to the HET templates, we obtained high-resolution $(R \sim 31,000)$ Echelle spectra using the ARCES instrument (Wang et al. 2003) on the APO 3.5m telescope. Seven exposures for a total of 63 minutes of integration were obtained. Data was reduced using a modified IRAF script originally written by J. Barentine and J. Krzesinski for ARCES data. Spectra are corrected for bias and dark subtraction, cosmic rays and bad pixels. Flatfielding is performed using a quartz lamp and two different sets of integration times: a "blue" set of 4 min integrations using a blue filter and a "red" set of $7 \mathrm{~s}$ integrations with no filter in the beam. These sets are then combined to form a master flatfield image. The two different quartz sets are used to maximize the signal-to-noise in both the blue and red end of the spectrum. Spectra are wavelength calibrated using a sequence of 10-s ThAr integrations taken a few times during each night. The star HD 42088 (spectral type 06V) was observed to remove telluric lines. 


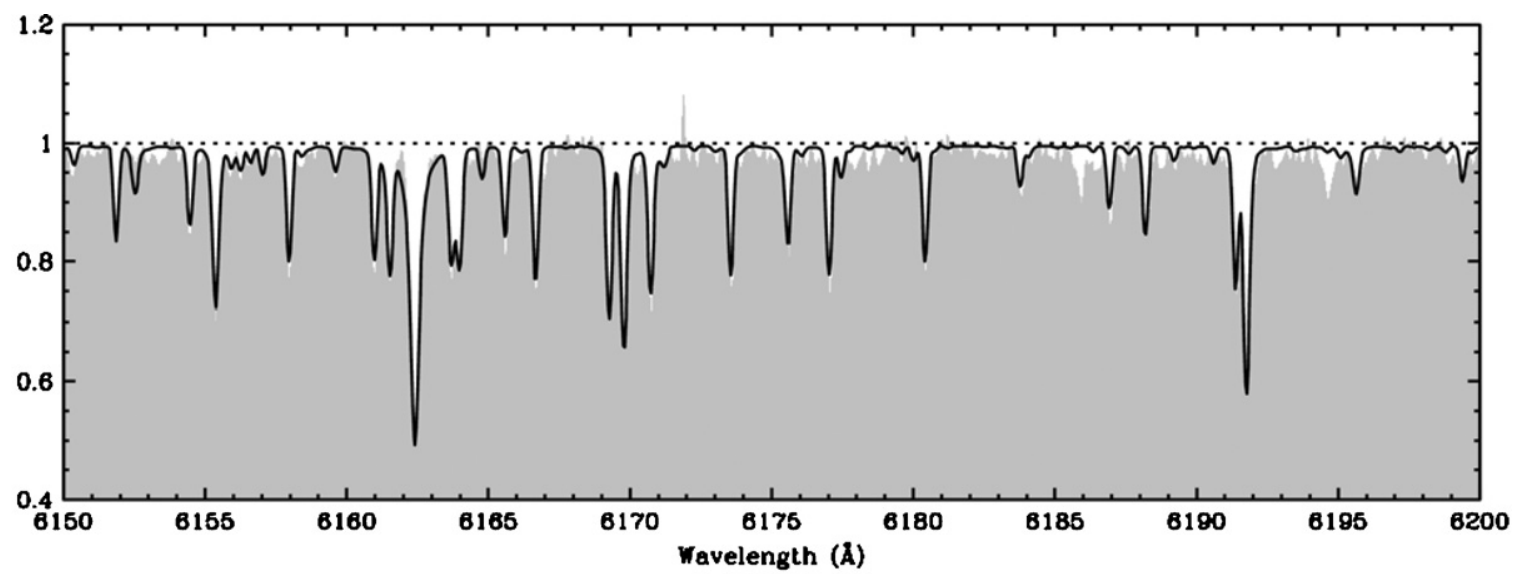

Figure 9. Portion of the ARCES spectrum $(R \sim 31,000)$ used in the SME analysis. The input spectrum is in white with the best-fit model overlaid in black.

The spectrum was analyzed using the IDL-based program Spectroscopy Made Easy, or SME (Valenti \& Piskunov 1996). This code uses synthetic spectra and multidimensional leastsquares minimization to determine the best set of stellar parameters for an observed spectrum. These parameters include effective temperature, surface gravity, metallicity, microturbulence, macroturbulence, projected rotational velocity, and the $\mathrm{RV}$. We follow the guidelines from previous spectroscopic studies of host stars that used SME (e.g., Valenti \& Fischer 2005; Stempels et al. 2007). We used three-dimensional interpolation on the Kurucz (1993) grid of local thermodynamic equilibrium (LTE) model atmospheres and the VALD database to obtain line data for transitions with predicted absorption cores deeper than $0.5 \%$ of the continuum. For the VALD queries, we used solar abundances, $T_{\text {eff }}=5770 \mathrm{~K}, \log g=4.44$, and $\xi_{t}=0.866 \mathrm{~km} \mathrm{~s}^{-1}$.

For the SME analysis, as suggested by Stempels et al. (2007), we fixed the parameter $\xi_{t}$ to $0.85 \mathrm{~km} \mathrm{~s}^{-1}$, in order to decouple the correlation between microturbulence $\xi_{t}$ and metallicity. For the macroturbulence $\zeta_{t}$, we follow the empirical relation of Valenti $\&$ Fischer (2005) which gives $\zeta_{t}=4.5 \mathrm{~km} \mathrm{~s}^{-1}$ for a star with $T_{\text {eff }} \sim 6200 \mathrm{~K}$. We were unable to obtain consistent results from the gravity-sensitive Mgb triplet region, therefore we fix $\log g$ at three values of $4.3,4.4$, and 4.5 , corresponding to the $1 \sigma$ range determined from the HET spectra. We set as free parameters $T_{\text {eff }}$, $[\mathrm{M} / \mathrm{H}], v \sin I$, and the $\mathrm{RV} v_{\text {rad }}$ and utilize the metal-rich region of 6000-6200 ̊. The uncertainties of the parameters are derived from the range of best-fit results using the three fixed $\log g$ values. We derive $T_{\text {eff }}=6246_{-45}^{+27} \mathrm{~K},[\mathrm{M} / \mathrm{H}]=0.3615_{-0.027}^{+0.009}$ and $v \sin I=7 \pm 1 \mathrm{~km} \mathrm{~s}^{-1}$, in reasonable agreement with the values derived from the HET spectra. Figure 9 shows the best-fit model in black and the input spectrum in white for a portion of the 6000-6200 A range used in the fitting.

The SME analysis of the ARCES spectrum is used as an independent check on the derived temperature and metallicity from the HET spectra. Because it is based on a smaller wavelength region, and cannot be used to independently derive the surface gravity, we chose to adopt the results from the HET analysis for the final stellar properties.

\section{DETERMINATION OF HOST STAR MASS AND RADIUS}

An alternative to interpolating isochrone models to determine stellar properties is to apply the analytical equations derived by Torres et al. (2010) using measurements of eclipsing binary

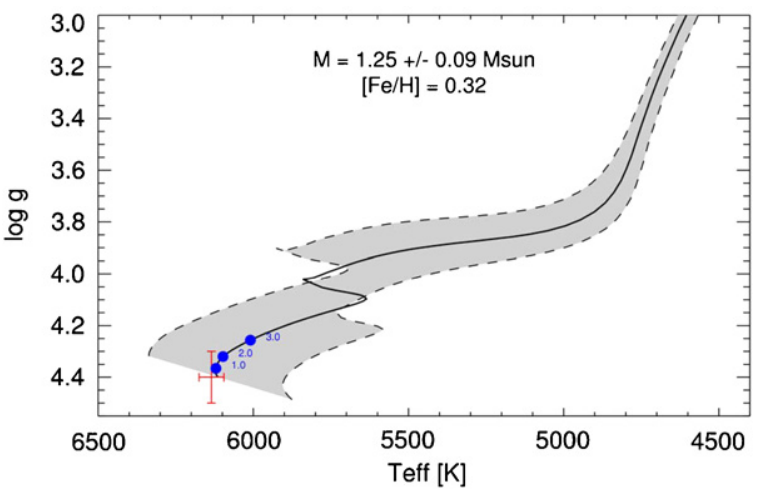

Figure 10. HR diagram as a function of $T_{\text {eff }}$ and $\log g$ based on Yonsei-Yale stellar evolution models. The solid track is for the best-fit stellar parameters of $1.25 M_{\odot}$ and $[\mathrm{Fe} / \mathrm{H}]=+0.32$. The two dashed tracks are for masses of $1.25 \pm 0.09 M_{\odot}$ and represent the $1 \sigma$ uncertainties on the mass. Blue dots are the location of the star at ages of $\{1,2,3\}$ Gyr, respectively. TYC 2949-00557-1 is consistent with a ZAMS star that has an age no older than $\sim 2$ Gyr.

(A color version of this figure is available in the online journal.)

systems. We use this empirical relation to derive the mass and radius of the primary using the values of $T_{\text {eff }}, \log g$ and $[\mathrm{Fe} / \mathrm{H}]$ obtained from the HET template spectra. Applying the equations for $\log M$ and $\log R$ yields a mass of $M=1.25 \pm 0.09 M_{\odot}$ and a radius of $R=1.15 \pm 0.15 R_{\odot}$. Correlations of the best-fit coefficients from Torres et al. (2010) are included in the errors, but correlations of $T_{\text {eff }}, \log g$ and $[\mathrm{Fe} / \mathrm{H}]$ are not considered. The reported scatter in the relation as found in Torres et al. (2010) of $\sigma_{\operatorname{logm}}=0.027$ and $\sigma_{\text {logr }}=0.014$ are also included in the mass and radius uncertainties, respectively, by adding them in quadrature.

Figure 10 compares the spectroscopically measured $T_{\text {eff }}$ and $\log g$ of TYC 2949-00557-1 (red error bars) to a theoretical stellar evolutionary track from the Yonsei-Yale ("Y ${ }^{2}$ ") model grid (see Demarque et al. 2004, and references therein). The solid curve represents the evolution of a single star of mass $1.25 M_{\odot}$ and metallicity of $[\mathrm{Fe} / \mathrm{H}]=+0.32$, starting from the zero-age main sequence (lower left corner), across the Hertzsprung gap, and to the base of the red giant branch. Symbols indicate various ages along the track labeled in Gyr. The dashed curves represent the same evolutionary track but for masses $\pm 0.09 M_{\odot}$, representative of the $1 \sigma$ uncertainty in the mass from the Torres et al. (2010) relation. The filled gray region between the two mass tracks represents the range of expected locations of a 
star like TYC 2949-00557-1 given the $1 \sigma$ mass uncertainty and measured metallicity. We emphasize that we have not directly measured the mass of TYC 2949 but rather derived the mass using the empirical relation of Torres et al. (2010). Our purpose here is not to test the accuracy of the theoretical stellar evolutionary tracks, but rather to constrain the evolutionary status of the TYC 2949 system. The spectroscopically measured $T_{\text {eff }}, \log g$, and $[\mathrm{Fe} / \mathrm{H}]$ place TYC 2949 near the zero-age main sequence, with an age of at most $\sim 2$ Gyr.

The distance to the host star can be computed once the bolometric luminosity is known. We use the Stefan-Boltzmann law to derive the luminosity of the star using the $T_{\text {eff }}$ found in Section 5.2 and the radius calculated by the Torres et al. (2010) relation. The absolute $V$ magnitude is then given by

$$
M_{V}=-2.5 \log \frac{L}{L_{\odot}}+M_{\mathrm{Bol}_{\odot}}-B C_{V}
$$

where $M_{\mathrm{Bol}_{\odot}}$ is the bolometric absolute magnitude of the Sun, here assumed to be 4.74, and $B C_{V}$ is the bolometric correction to the $V$ band. We adopt a value of $B C_{V}=-0.175$, interpolated from Table 15.7 in Drilling \& Landolt (2004) based on the $T_{\text {eff }}$ of TYC 2949-00557-1. The distance can then be calculated via the distance modulus and assuming a value for the lineof-sight extinction. If we assume $A_{V}=0.45 \pm 0.1$, which is consistent with both the Schlegel et al. (1998) dust maps and the best-fit value obtained from the SED fitting in Section 5.1, we derive a distance to the system of $413_{-57}^{+66} \mathrm{pc}$. This distance is consistent with our implicit assumption that the star is behind the majority of the dust along this line of sight, for likely values of the thickness of the dust layer. The quoted uncertainty in the distance includes the uncertainties in the stellar radius, effective temperature, line-of-sight extinction and apparent $V$-band magnitude.

\section{COMPANION MASS}

Given the orbital parameters from the joint RV fit (Section 2.4), and the estimate of the mass of the host star derived from the spectroscopic parameters (Section 5.2), we can estimate a minimum mass for the companion of

$$
m_{\min }=64.3 \pm 3.0 M_{J},
$$

where we have assumed a circular orbit. This minimum mass is based only on the RV and stellar parameters, and ignores the fact that edge-on configurations are likely excluded given the lack of transit signature. Since the minimum mass is below the hydrogen burning limit $\left(\sim 80 M_{J}\right)$, one might be tempted to categorize this object as a brown dwarf. However, since the orbital inclination $i$ is unknown, we do not know the true mass, and given that the minimum mass is not far below the hydrogen burning limit, it does not suffice to make the usual assumption that the minimum mass of the object can be used to characterize its nature. Rather, we must be somewhat more careful to estimate the probability distribution for its true mass.

We proceed to estimate the probability distribution of the companion mass using a similar method as was used to search for and exclude transits in the KELT photometric data as described in Section 4. We use the distribution of companion periods $P$, and velocity semi-amplitudes $K$ from the Markov chain derived from the MCMC analysis of the combined RV data described in Section 2.4, for the fit assuming zero eccentricity. For each set of these parameters (each link in the chain), we draw a random

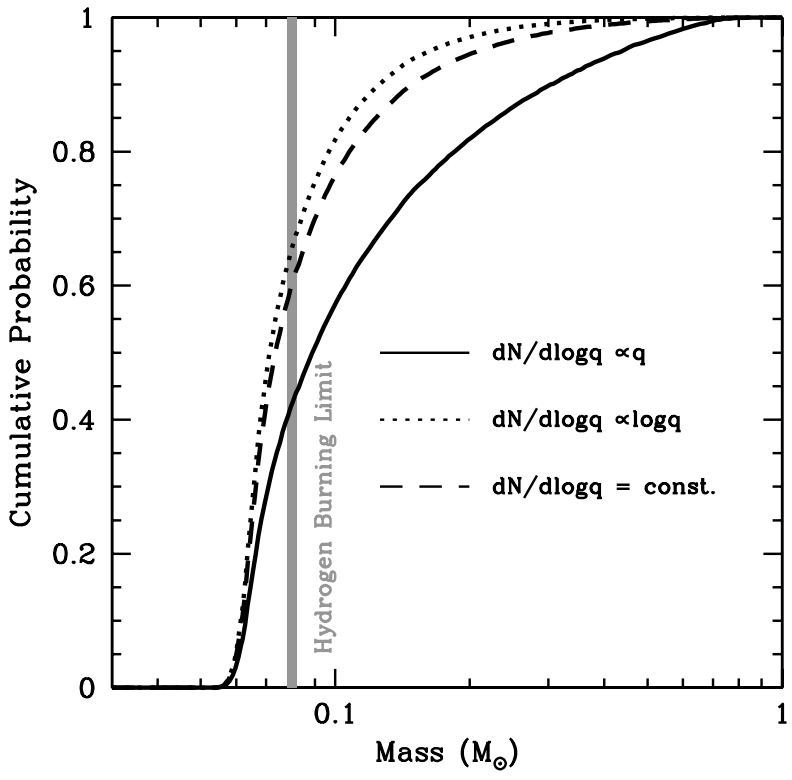

Figure 11. Cumulative probability that the mass of the companion to TYC 294900557-1 is less than a given mass in solar masses. These probabilities account for the uncertainties and covariances between the parameters of the RV fit, the uncertainty in the mass of the primary, assuming a uniform distribution of $\cos i$, and adopting various priors for the distribution of companion mass ratios $d N / d \log q$.

value for the spectroscopically determined primary parameters $T_{\text {eff }}, \log g$, and $[\mathrm{Fe} / \mathrm{H}]$, according to a Gaussian distribution centered on the best-fit values and with dispersions equal to the uncertainties (given in Table 5). We use these values of $T_{\text {eff }}$, $\log g$, and $[\mathrm{Fe} / \mathrm{H}]$ to estimate the primary mass $M$ using the empirical relation of Torres et al. (2010). We add an additional offset to $M$ drawn from a Gaussian with dispersion equal to the dispersion in the fit to this empirical relation (6.4\%). We draw a random value of $\cos i$ from a uniform distribution in the range $(0,1)$, and then solve for the mass $m$ of the secondary (note we do not assume that $m \ll M)$.

We weight the resulting distribution of $m$ by a prior on the luminosity ratio $l$ and a prior on the mass ratio $q$. Specifically, we assume luminosity ratios of $l \gtrsim 0.1$ are excluded by the lack of features due to the companion in the high-resolution spectra. We assume a flux ratio relationship of the form $l \propto q^{4.5}$, as is roughly appropriate for main-sequence stars. The exponent of 4.5 is derived by fitting the values found in Table 1 of Torres et al. (2010) for stars with $0.5<M_{\odot}<1.5$. We note that the precise value of the exponent for this relationship does not significantly affect our conclusions for reasonable values in the range 2.5-6.5. We therefore assume the companion is on the main sequence and is not a remnant. Since the mass ratio distribution for companions in the relevant range of masses is uncertain, we adopt three different priors that are likely to bracket the true distribution (see, e.g., Duquennoy \& Mayor 1991; Mazeh et al. 1992; Grether \& Lineweaver 2006). Specifically, we assume linear $(d N / d \log q \propto q)$, logarithmic $(d N / d \log q \propto \log q)$, and constant $(d N / d \log q=$ constant $)$ priors. For the logarithmic and constant priors, a brown dwarf companion is slightly favored ( $\sim 66 \%$ and $61 \%$, respectively), whereas for the linear prior, a stellar companion is slightly favored (57\%). Figure 11 plots the cumulative probability that the mass of the companion is less than a given mass for the three different assumed priors. 


\section{EFFECTS OF TIDES}

Given the proximity of the companion to the host star, tidal interactions could be important in this system. The tidal effects between solar mass stars and very low mass stars/brown dwarfs are not well studied. Moreover, tidal models themselves are complicated and uncertain, and observational constraints are few, especially among brown dwarfs and very late $M$ dwarfs. The situation is further complicated by the ambiguity of the secondary's mass, which partly determines the tidal evolution. Nonetheless, we consider the tidal evolution of the system in this section.

Following Goldreich \& Soter (1966), we assume that the tidal response of a body can be adequately modeled by a single parameter, $Q^{\prime}$, which is related to the angle between the lines connecting the centers of the two bodies, and the center of the deformed body to its tidal bulge. See Heller et al. (2010) or Ferraz-Mello et al. (2008) for recent reviews of tidal theory. Furthermore, we require that as the orbit of the secondary evolves, the parameter $Q^{\prime}$ remains constant. If we assume the orbit is circular, it may be shown that the semi-major axis of the secondary decays as

$$
\frac{d a}{d t}=\frac{9}{2} \sqrt{\frac{G}{M}} \frac{R^{5} m}{Q_{*}^{\prime}} a^{-11 / 2}
$$

(Goldreich \& Soter 1966), where $Q_{*}^{\prime}$ is the tidal quality factor of the star divided by two-thirds of its Love number, $M$ is the mass of the primary, $R$ is the radius of the primary and $m$ is the mass of the secondary. Subscript "*" refers to the primary.

Equation (5) only applies if the primary's rotation period is longer than the orbital period. Should the two be equal, the tidal bulges align, the torques reduce to zero, and the orbital evolution effectively halts. There still may be evolution due to the obliquity tide raised on the star, but that evolution is orders of magnitude slower, and we ignore it here. As the secondary decays, its angular momentum, $L_{\text {orb }}=m \sqrt{G M a}$, decreases and is passed to the star's rotational angular momentum, $L_{*}=C_{*} M R^{2} \Omega$, where $\Omega$ is the primary's rotation frequency, and $C_{*}$ is the primary's moment of inertia coefficient. We set $C_{*}$ to 0.1 (Massarotti 2008). Therefore, over a given time, the change in the primary's rotational frequency is

$$
\Delta \Omega=-\frac{\Delta L_{\mathrm{orb}}}{C_{*} M R_{*}^{2}}
$$

where $\Delta L_{\text {orb }}$ is the change in orbital angular momentum over the same time. Equations (5) and (6) can be solved together to determine when the rotational frequency equals the orbital frequency (i.e., synchronization) when orbital decay stops. Here we ignore the case of the primary's rotation period being shorter than the orbital period, but in that case, the secondary will spin down the star, while its orbit expands, also driving the system toward synchronization.

We have examined the tidal evolution of this system in the range $10^{4} \leqslant Q_{*}^{\prime} \leqslant 10^{10}$. Most studies find $Q_{*}^{\prime}$ values in the range $10^{5}-10^{7}$ (Mathieu 1994; Jackson et al. 2008; but see also Barker \& Ogilvie (2010)), but in reality this parameter is very poorly constrained. Our chosen range covers all plausible values. We also vary $i$, the inclination of the secondary's orbit to the line of sight, for $90^{\circ}-1^{\circ}$ (from edge-on to nearly face-on) and adjust the mass accordingly. We determine the primary's rotation period via the measured value of $v \sin I$ and the derived radius of the primary, $R$. For this analysis, we set the primary's equator in

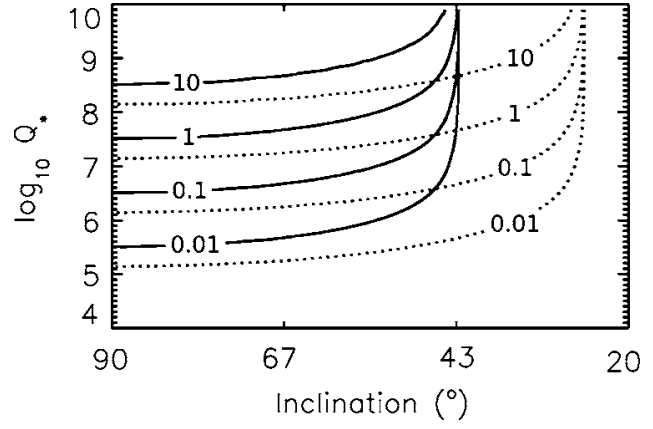

Figure 12. Contours of the time required to synchronize the primary's rotational period to the orbital period in Gyr. Solid contours correspond to the best fit, dotted contours are for an unlikely but plausible case which maximizes the synchronization timescale.

the same plane as the secondary's orbit (i.e., we assume $i=I$ ), but this decision does not qualitatively affect our results.

In Figure 12 we show the synchronization times from Equations (5)-(6) over the parameter space defined above. We consider two models, the best-fit set of parameters (solid contours), and one in which $v \sin I=4 \mathrm{~km} \mathrm{~s}^{-1}$ (the 3-sigma minimum value), $M=1.22 M_{\odot}$, and $R=1.02 R_{\odot}$ (dotted contours), i.e., a pathological case, permitted by the observational uncertainties, with values of $v \sin I, M$, and $R$ that minimize the tidal evolution. First, note the convergence of contour lines at $i=43^{\circ}$ ( solid contours) and $i=25^{\circ}$ (dotted contours). These singularities occur because $i$ is small enough that the system is currently synchronized, i.e., $v \sin I$ equals the circular velocity of the companion. For $i$ greater than these values the secondary is spinning up the primary. We find that for a wide range of $Q_{*}^{\prime}$ and $i$ combinations, the secondary quickly spins the primary up to synchronization. For the best fit and $Q_{*}^{\prime} \lesssim 10^{7}$, the synchronization time is $\lesssim 0.1$ Gyr. The photometry shows no significant variability due to starspots that would indicate a particularly young F star, so it is likely that the age of the system is larger than the synchronization timescale. We interpret this short timescale as evidence that the secondary has already spun the primary up to synchronization. For the pathological case, the time to synchronize could be a factor of a few larger, but still small compared to the likely age of the system. We do not show the analogous case for large $v \sin I, M$, and $R$, as that case is already synchronized for all values of $i$.

We conclude that this system is most likely in a double synchronous state in which the orbit is circular, and both primary and secondary rotational frequencies are equal to the orbital frequency. We emphasize that this conclusion is tentative; should more information regarding $Q_{*}^{\prime}$ or the relative orientations of the primary's spin axis and orbital plane be determined, our analysis will need to be updated.

\section{CONCLUSION}

We have discovered a brown dwarf candidate around the main sequence F star TYC 2949-00557-1 during the MARVELS pilot project, a wide-area, multi-object, RV search for planets. We have characterized the properties of the host star and the dynamics of the orbiting companion. The companion straddles the hydrogen burning minimum mass with an $m_{\min } i=64 M_{J}$, and the orbital period of $\sim 5.7$ days places this candidate in the brown dwarf "orbital separation desert." This desert extends to very low-mass stars (VLMS) with masses up to $\sim 150 \mathrm{M}_{\mathrm{J}}$, reflective of the fact that the formation and dynamical evolution 
of these objects is independent of the precise substellar mass limit. Therefore, TYC 2949-00557-1b is still a "desert dweller" for all but the most extreme face-on inclinations. Tidal analysis suggests that the system is in a double synchronous state where both companions have achieved rotational synchronization in addition to achieving orbital synchronization.

If the companion's true mass is substellar, this is an example of a rare class of brown dwarfs at orbital distances comparable to those of "Hot Jupiters." Our photometric observations show no evidence of variability. Although we are able to exclude transits for likely companion radii, the orbital inclination is otherwise unconstrained, and thus there remains the possibility that this system is a VLMS companion with a nearly face-on inclination. Depending on the assumed priors for the distribution of binary mass ratios, we estimate a probability of $43 \%-66 \%$ that the companion is below the hydrogen burning minimum mass. The large number of stars surveyed by multi-object, $\mathrm{RV}$ instruments may allow for additional rare systems to be discovered, including brown dwarfs in the mass or orbital separation deserts. Such rare systems can provide important constraints to models of the formation and internal structure of low-mass stars and brown dwarfs, in addition to elucidating the formation and evolution of extrasolar planetary systems.

We thank the anonymous referee for comments that improved the quality of this paper. Thanks to Eric Agol for editing of the manuscript draft that greatly improved the quality of the paper. Funding for the multi-object Doppler instrument was provided by the W.M. Keck Foundation. The pilot survey was funded by NSF with grant AST-0705139, NASA with grant NNX07AP14G and the University of Florida. S.W.F. is supported through a Florida Space Grant Fellowship. K.G.S, and J.P. acknowledge funding support from the Vanderbilt Initiative in Data-Intensive Astrophysics (VIDA) from Vanderbilt University, and from NSF Career award AST-0349075. This research has made use of the SIMBAD database, operated at CDS, Strasbourg, France. Based on observations with the SDSS $2.5 \mathrm{~m}$ telescope. Funding for the SDSS and SDSS-II has been provided by the Alfred P. Sloan Foundation, the Participating Institutions, the National Science Foundation, the U.S. Department of Energy, the National Aeronautics and Space Administration, the Japanese Monbukagakusho, the Max Planck Society, and the Higher Education Funding Council for England. The SDSS Web Site is http://www.sdss.org/. The SDSS is managed by the Astrophysical Research Consortium for the Participating Institutions. The Participating Institutions are the American Museum of Natural History, Astrophysical Institute Potsdam, University of Basel, University of Cambridge, Case Western Reserve University, University of Chicago, Drexel University, Fermilab, the Institute for Advanced Study, the Japan Participation Group, Johns Hopkins University, the Joint Institute for Nuclear Astrophysics, the Kavli Institute for Particle Astrophysics and Cosmology, the Korean Scientist Group, the Chinese Academy of Sciences (LAMOST), Los Alamos National Laboratory, the Max-PlanckInstitute for Astronomy (MPIA), the Max-Planck-Institute for Astrophysics (MPA), New Mexico State University, Ohio State University, University of Pittsburgh, University of Portsmouth, Princeton University, the United States Naval Observatory, and the University of Washington. Based on observations obtained with the Apache Point Observatory $3.5 \mathrm{~m}$ telescope, which is owned and operated by the Astrophysical Research Consortium. This publication makes use of data products from the Two Micron All Sky Survey, which is a joint project of the
University of Massachusetts and the Infrared Processing and Analysis Center/California Institute of Technology, funded by the National Aeronautics and Space Administration and the National Science Foundation. The Hobby-Eberly Telescope (HET) is a joint project of the University of Texas at Austin, Pennsylvania State University, Stanford University, Ludwig-Maximillians-Universität München, and GeorgAugust-Universität Göttingen. The HET is named in honor of its principal benefactors, William P. Hobby and Robert E. Eberly. The Center for Exoplanets and Habitable Worlds is supported by the Pennsylvania State University, the Eberly College of Science, and the Pennsylvania Space Grant Consortium.

\section{REFERENCES}

Alard, C., \& Lupton, R. H. 1998, ApJ, 503, 325

Alvarez, R., \& Plez, B. 1998, A\&A, 330, 1109

Armitage, P. J., \& Bonnell, I. A. 2002, MNRAS, 330, 11

Asplund, M. 2005, ARA\&A, 43, 481

Baraffe, I., Chabrier, G., Barman, T. S., Allard, F., \& Hauschildt, P. H. 2003, A\&A, 402, 701

Barker, A. J., \& Ogilvie, G. I. 2010, MNRAS, 404, 1849

Barklem, P. S., \& O’Mara, B. J. 1998, MNRAS, 300, 863

Burke, C. J., Gaudi, B. S., DePoy, D. L., \& Pogge, R. W. 2006, AJ, 132, 210

Claret, A. 2000, A\&A, 363, 1081

Cumming, A., Marcy, G. W., \& Butler, R. P. 1999, ApJ, 526, 890

Drilling, J. S., \& Landolt, A. U. 2004, in Allen's Astrophysical Quantities, ed. A. N. Cox (New York: Springer), 388

Deleuil, M., et al. 2008, A\&A, 491, 889

Demarque, P., Woo, J.-H., Kim, Y.-C., \& Yi, S. K. 2004, ApJS, 155, 667

Duquennoy, A., \& Mayor, M. 1991, A\&A, 248, 485

Endl, M., Hatzes, A. P., Cochran, W. D., McArthur, B., Allende Prieto, C., Paulson, D. B., Guenther, E., \& Bedalov, A. 2004, ApJ, 611, 1121

Erskine, D. J. 2002, PASP, 115, 255

Erskine, D. J., Edelstein, J., Feuerstein, W. M., \& Welsh, B. 2003, ApJ, 592, 103

Ferraz-Mello, S., Rodríguez, A., \& Hussman, H. 2008, Celest. Mech. Dyn. Astron., 101, 171

Ford, E. B. 2006, ApJ, 642, 505

Ge, J. 2002, ApJ, 571, L165

Ge, J., Erskine, D. J., \& Rushford, M. 2002, PASP, 114, 1016

Ge, J., et al. 2006a, Proc. SPIE, 6269, 75

Ge, J., et al. 2006b, ApJ, 648, 683

Ge, J., et al. 2009, Proc. SPIE, 7440, 18

Goldreich, P., \& Soter, S. 1966, Icarus, 5, 375

Grether, D., \& Lineweaver, C. H. 2006, ApJ, 640, 1051

Gunn, J. E., et al. 2006, AJ, 131, 2332

Gustafsson, B., Edvardsson, B., Eriksson, K., Jørgensen, U. G., Nordlund, Å., \& Plez, B. 2008, A\&A, 486, 951

Hartman, J. D., Gaudi, B. S., Holman, M. J., McLeod, B. A., Stanek, K. Z., Barranco, J. A., Pinsonneault, M. H., \& Kalirai, J. S. 2008, ApJ, 675, 1254 Hauschildt, P. H., Allard, F., \& Baron, E. 1999, ApJ, 512, 377

Heller, R., Jackson, B., Barnes, R., Greenberg, R., \& Homeier, D. 2010, A\&A, 514,22

Høg, E., et al. 2000, A\&A, 355L, 27

Jackson, B., Greenberg, R., \& Barnes, R. 2008, ApJ, 678, 1396

Jenkins, J. S., et al. 2009, MNRAS, 398, 911

Kane, S. R., Schneider, D. P., \& Ge, J. 2007, MNRAS, 377, 1610

Kane, S. R., Mahadevan, S., Cochran, W. D., Street, R. A., Thirupathi, S., Henry, G. W., \& Williamson, M. H. 2009, ApJ, 692, 290

Kovács, G., Bakos, G., \& Noyes, R. W. 2005, MNRAS, 356, 557

Kupka, F., Piskunov, N., Ryabchikova, T. A., Stempels, H. C., \& Weiss, W. W. 1999, A\&AS, 138, 119

Kurucz, R. 1993, ATLAS9 Stellar Atmosphere Programs and $2 \mathrm{~km} \mathrm{~s}^{-1}$ Grid, Kurucz CD-ROM No. 13 (Cambridge, MA: Smithsonian Astrophysical Observatory)

Lomb, N. R. 1976, Ap\&SS, 39, 447

Mahadevan, S., van Eyken, J., Ge, J., DeWitt, C., Fleming, S. W., Cohen, R., Crepp, J., \& Vanden Heuvel, A. 2008, ApJ, 678, 1505

Mandel, K., \& Agol, E. 2002, ApJ, 580, L171

Marcy, G. W., \& Butler, R. P. 2000, PASP, 112, 137

Marcy, G. W., et al. 2001, ApJ, 555, 418

Massarotti, A. 2008, AJ, 135, 2287

Mathieu, R. 1994, ARA\&A, 32, 465 
Matzner, C. D., \& Levin, Y. 2005, ApJ, 628, 817

Mazeh, T. 2008, in , Observational Evidence for Tidal Interactions in Close Binary Systems, ed. M.-J. Groupil \& J.-P. Zahn (EAS Publications Ser. 29; Paris: EDP Sciences), 1

Mazeh, T., Goldberg, D., Duquennoy, A., \& Mayor, M. 1992, ApJ, 401, 265

Metchev, S. A., \& Hillenbrand, L. A. 2009, ApJS, 181, 62

Niedzielski, A., Nowak, G., Adamów, M., \& Wolszczan, A. 2009, ApJ, 707, 768

Omiya, M., et al. 2009, PASJ, 61, 825

Patel, S. G., Vogt, S. S., Marcy, G. W., Johnson, J. A., Fischer, D. A., Wright, J. T., \& Butler, R. P. 2007, ApJ, 665, 744

Pfahl, E., Arras, P., \& Paxton, B. 2008, ApJ, 679, 783

Pepper, J., Gould, A., \& Depoy, D. L. 2003, Acta Astron., 53, 213

Pepper, J., et al. 2007, PASP, 119, 923

Plez, B., \& Cohen, J. G. 2005, A\&A, 434, 1117

Pont, F. 2009, MNRAS, 396, 1789

Ramsey, L. W., et al. 1998, Proc. SPIE, 3352, 34

Santos, N. C., Israelian, G., \& Mayor, M. 2004, A\&A, 415, 1153

Santos, N. C., et al. 2002, A\&A, 392, 215

Scargle, J. D. 1982, ApJ, 263, 835

Schlegel, D. J., Finkbeiner, D. P., \& Davis, M. 1998, ApJ, 500, 525

Shetrone, M., et al. 2007, PASP, 119, 556
Siverd, R. J., Pepper, J., Stanek, K., Pogge, R. W., Gaudi, B. S., \& DePoy, D. L. 2009, in IAU Symp. 253, Transiting Planets, ed. F. Pont, D. Queloz, \& D. Sasselov (Cambridge: Cambridge Univ. Press), 350

Skrutskie, M. F., et al. 2006, AJ, 131, 1163

Smith, J. A., et al. 2002, AJ, 123, 2121

Stassun, K. G., Mathieu, R. D., \& Valenti, J. A. 2006, Nature, 440, 311

Stassun, K. G., Mathieu, R. D., \& Valenti, J. A. 2007, ApJ, 664, 1154

Stempels, H. C., Collier Cameron, A., Hebb, L., Smalley, B., \& Frandsen, S. 2007, MNRAS, 379, 773

Stetson, P. B. 1987, PASP, 99, 191

Torres, G., Andersen, J., \& Giménez, A. 2010, A\&AR, 18, 67

Tull, R. G. 1998, Proc. SPIE, 3355, 387

Udry, S., Mayor, M., Naef, D., Pepe, F., Queloz, D., Santos, N. C., \& Burnet, M. 2002, A\&A, 390, 267

Valenti, J., \& Fischer, D. 2005, ApJS, 159, 141

Valenti, J., \& Piskunov, N. 1996, A\&AS, 118, 595

van Eyken, J. C., Ge, J., Mahadevan, S., \& DeWitt, C. 2004, ApJ, 600, 79

van Eyken, J. C., Ge, J., \& Mahadevan, S. 2010, ApJS, in press (arXiv:1005.5564v1)

Wang, S., et al. 2003, Proc. SPIE, 4841, 1145

Wittenmyer, R. A., Endl, M., Cochran, W. D., Ramírez, I., Reffert, S., MacQueen, P. J., \& Shetrone, M. 2009, AJ, 137, 3529 